\title{
HOMOGENIZATION OF CONSTRAINED OPTIMAL CONTROL PROBLEMS FOR ONE-DIMENSIONAL ELLIPTIC EQUATIONS ON PERIODIC GRAPHS *
}

\author{
Peter I. Kogut ${ }^{1}$ And GÜnter Leugering ${ }^{2}$
}

\begin{abstract}
We are concerned with the asymptotic analysis of optimal control problems for 1-D partial differential equations defined on a periodic planar graph, as the period of the graph tends to zero. We focus on optimal control problems for elliptic equations with distributed and boundary controls. Using approaches of the theory of homogenization we show that the original problem on the periodic graph tends to a standard linear quadratic optimal control problem for a two-dimensional homogenized system, and its solution can be used as suboptimal controls for the original problem.
\end{abstract}

Mathematics Subject Classification. 35B27, 35J25, 49J20, 93C20.

Received January 7, 2005. Revised January 20, 2006 and December 8, 2006.

Published online June 24, 2008.

\section{INTRODUCTION}

In this paper we consider a linear-quadratic control-constrained optimal control problem for 1-D partial differential elliptic equations defined on a periodic planar graph (3.3), (3.4), (3.5), (3.6). We study the asymptotic behavior of this problem when the $\varepsilon$-period of the graph tends to zero, and look for the limiting homogenized optimal control problem. In particular, we require that an optimal solution and the minimum of the cost functional for the homogenized problem are the limit values (in a certain sense) of the corresponding quantities of the original problem. The solution to the homogenized constrained optimal control problem is shown to serve as a suboptimal control for $\epsilon$-level problem when restricted to the graph.

Even though partial differential equations on networked domains and their homogenized substitutes are very important in various mechanical, medical and industrial applications and constitute a growing field of interest, only few papers $[7,22-24,26,33]$ deal with the homogenization problem on periodic networks. Typically, not only the process on the graph itself but rather the optimization and control of processes on such networks are of great importance. On the one side, as one goes down the scales of the periodic structures, the numerical computation of the solution of these problems is very costly due to the singular behavior for small scales and the complexity of large networks, while on the other side local computations on a regular locally 1-d grid are much easier than 2-d or 3-d calculations. It may thus happen, as in sparse-grid computations, that an originally

\footnotetext{
Keywords and phrases. Optimal control, homogenization, elliptic equation, periodic graph.

* We gratefully acknowledge the support of the DAAD.

${ }^{1}$ Department of Differential Equations, Dnipropetrovsk National University, Naukova str., 13, 49050 Dnipropetrovsk, Ukraine. p.kogut@i.ua

${ }^{2}$ Institüt für Angewandte Mathematik Lehrstuhl II, Universität Erlangen-Nürnberg Martensstr.3, 91058 Erlangen, Germany. Guenter.Leugering@am. uni-erlangen.de
} 
2-d or 3-d problem is approximated by a semi-discretization on a suitable grid, whereas on the other side, as in carbon-nanotube technology, photonic lattice devices and also in infrastructural problems involving water and gas-networks, the vascular system, to mention just a few applications, a problem on a planar (or 3-d) graph may be substituted by a homogenized one on a simple but higher dimensional domain. Thus, asymptotic analysis for optimal control problems on graphs appears to be a major tool in order investigate these questions. Moreover, in such an asymptotic analysis we may require both the optimal solution and the minimal value of the cost functional for the original problem to converge to the corresponding characteristics of a limit optimal control problem, as $\varepsilon$ tends to zero.

It should be stressed that the original problem (on the graph) and the homogenized one (in a 2-d domain) live in the different function spaces. Moreover, if the small parameter $\varepsilon$ is changed, then all components of the original control problem, including the $\varepsilon$-periodic graph $\Omega_{\varepsilon}$, the control constraint sets, the cost functional, and the set, where we seek its infimum, are changed as well. Let us observe also that the Lebesgue measure of the "material" included in the periodic graph $\Omega_{\varepsilon}$ is equal to zero for every $\varepsilon>0$, whereas there exists a set $\Omega$ which is filled up by this planar graph in the limit, as $\varepsilon \rightarrow 0$. In view of this, our approach is based on the description of boundary value problems for planar networks in terms of singular measures as proposed by Zhikov, Bouchitte, and Fragala in their recent works [3,8,34,35]. Our emphasis is not on the homogenization of the underlying system of partial differential equations on graphs as such, but rather on the homogenization of the optimal control problem with distributed controls and Neumann boundary controls on planar $\varepsilon$-periodic graphs as $\varepsilon \rightarrow 0$.

For the asymptotic analysis of optimal control problems in general we refer to e.g. $[1,6,10,13,14,27,30]$. The most typical procedure of homogenization consists of the following steps: at first, we write down the necessary optimality conditions for the initial problem; next we find the corresponding limiting relations as $\varepsilon \rightarrow 0$ and interpret them as necessary optimality conditions for some control problem; then, using the limiting necessary optimality conditions, we recover an optimal control problem which is called the homogenized control problem (see e.g. $[2,11,15,16,32]$ ). Thus, if we denote by $\mathrm{OCP}_{\varepsilon}, \mathrm{NOC}_{\varepsilon}, \mathrm{HOCP}, \mathrm{HNOC}$ the original optimal control problem on the $\varepsilon$-level, the corresponding necessary optimality conditions on the $\varepsilon$-level, the homogenized optimal control problem and the homogenized necessary optimality system, respectively, then the above mentioned procedure can be represented in the following diagram:

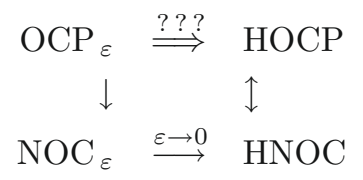

However, this diagram may not commute. Moreover, it should be stressed that the approach above is suitable only for simple enough (from the point of view of control theory) optimal control problems for which there are no restrictions on admissible pairs and their optimality conditions satisfy some regularity property [25]. An attempt to extend this approach to wider class of optimal control problems was realized in [11], where it was shown that the recovery of the homogenized optimal control problem is possible only under some additional assumptions on the structure of the state equation and the dependence on the small parameter.

We propose another approach to the homogenization of optimal boundary control problems, which is based on ideas in $\Gamma$-convergence and the concept of variational convergence of constrained minimization problems [4$6,30]$. To investigate the asymptotic behavior of the considered optimal boundary control problem we apply the scheme of direct homogenization, which was developed in [17-21]. Such approach allows to reduce the procedure of the homogenization to the consecutive identification of the set of admissible solutions for the homogenized optimal control problem and then its cost functional.

The plan of our paper is as follows. In Section 2 the main notion concerning $\varepsilon$-periodic graph-like structures in $\mathbb{R}^{2}$, the description of the geometry of periodic graph-structures and their boundary in terms of singular measures are given. It is important to note that we introduce two types of singular measures. One of them we use for the representation of $\varepsilon$-periodic bounded graph, and the second one is a 'boundary'-measure for 


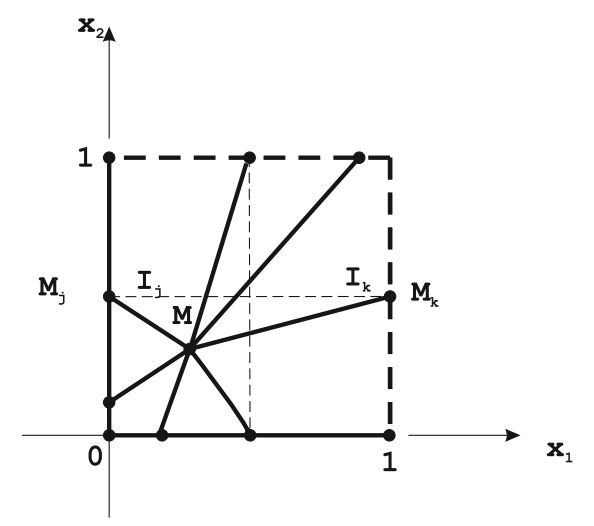

Figure 1. The cell of periodicity.

the description of boundary conditions on graph-like domain. In particular, we prove that sequences of the corresponding scaling measures weakly converge to some Lebesgue measures.

In Section 3 we briefly describe the main results originally due to Zhikov concerning to the construction of Sobolev spaces on graphs. The statement of optimal boundary control problem for one-dimensional elliptic equations on periodic graphs we give in Section 4. The results concerning to the extension of two-scale convergence with respect to singular measure, and the notion of the weak convergence (or shortly, w-convergence) of sequences of triplets: 'state-distributed control-boundary control' we cite in Section 5. Here we also prove a $w$-compactness result for the sequences of admissible solutions.

Section 6 contains the main homogenization results, where it is shown that the control constrained optimal control problem on the periodic graph converges to a control-constrained optimal control problem in a planar domain. In Section 7 we show that an optimal solution to the limit problem can be used as basis for the construction of suboptimal controls for the original control problem. In a $\delta$-neighborhood of the solution of the homogenized optimal control problem we find smooth controls, the traces of which along the $\varepsilon_{\text {-graph }} \Omega_{\varepsilon}$ are approximations to the optimal controls on $\Omega_{\varepsilon}$. In the last section we consider, as an example, the homogenization procedure for an optimal control problem on $\varepsilon$-periodic 'cross-like' graphs.

\section{2. $\varepsilon$-PERIODIC GRAPH-LIKE STRUCTURES IN $\mathbb{R}^{2}$ AND THEIR DESCRIPTION}

We begin with some notation. We say that the set $\square=[0,1)^{2}$ is the cell of periodicity for some planar graph $\mathcal{F}$ on $\mathbb{R}^{2}$ if $\square$ contains a "star"-structure such that (see Fig. 1): (i) all edges of this structure have a common point $M \in$ int $\square$; (ii) each edge is a line-segment and all end-points of these edges belong to the boundary of $\square$; (iii) in the set of end-points (vertices) there exist pairs $\left(M_{i} ; M_{k}\right)$ such that $x_{1}^{M_{i}}=x_{1}^{M_{k}}$ or $x_{2}^{M_{i}}=x_{2}^{M_{k}}$ (so, we admit the existence of isolated vertices). Let $\varepsilon \in E=\left(0, \varepsilon^{0}\right]$ be a small parameter, such that $\varepsilon$ varies in a strictly decreasing sequence of positive numbers which converges to 0 . We define the periodic graph $\mathcal{F}_{\varepsilon}:=\varepsilon \mathcal{F}=\{\varepsilon x: x \in \mathcal{F}\}$. Moreover, let $\square^{S}=\left\{\left(x_{1}, x_{2}\right): x_{1} \in[0,1), x_{2}=0\right\}, I^{\text {ed }}=\left\{I_{j}, j=1,2, \ldots, K\right\}$ be the set of all edges on $\square$, and let $\mathcal{M}=\left\{M_{i}, i=1,2, \ldots, L\right\}$ be the set of all vertices on $\square$ which belong to $\square^{S}$. Let $\Omega$ be an open bounded domain in $\mathbb{R}^{2}$ such that

$$
\Omega=\left\{\left(x_{1}, x_{2}\right): x_{1} \in \Gamma_{1}, 0<x_{2}<\gamma\left(x_{1}\right)\right\}
$$

where $\Gamma_{1}=(0, a), \gamma \in \mathrm{C}^{1}([0, a])$, and $0<\gamma_{0}=\inf _{x_{1} \in[0, a]} \gamma\left(x_{1}\right)$. It is clear that in this case $\partial \Omega=\Gamma_{1} \cup \Gamma_{2}$, where $\Gamma_{2}=\partial \Omega \backslash \Gamma_{1}$. We then define $\Omega_{\varepsilon}=\Omega \cap \mathcal{F}_{\varepsilon}$.

Following Zhikov's approach (see [34,35]) we describe the geometry of the set $\Omega_{\varepsilon}$ in terms of so-called singular measures in $\mathbb{R}^{2}$. To this end, for every segment $I_{i} \in I^{\mathrm{ed}}, i=1,2, \ldots, K$, we denote by $\mu_{i}$ its corresponding 
Lebesgue measure. Now we define the $\square$-periodic Borel measure $\mu$ in $\mathbb{R}^{2}$ as follows

$$
\mu=\sum_{i=1}^{K} g_{i} \cdot \mu_{i}
$$

where $g_{1}, g_{2}, \ldots, g_{K}$ are non-negative weights such that $\int \mathrm{d} \mu=1$. Thus the support of the measure $\mu$ is the union of all edges $I_{i} \in I^{\text {ed }}$. Since the homothetic contraction of the plane at $\varepsilon^{-1}$ takes the grid $\mathcal{F}$ to $\mathcal{F}_{\varepsilon}=\varepsilon \mathcal{F}$, we introduce a "scaling" $\varepsilon \square$-periodic measure $\mu_{\varepsilon}$ as follows

$$
\mu_{\varepsilon}(B)=\varepsilon^{2} \mu\left(\varepsilon^{-1} B\right) \text { for every Borel set } B \subset \mathbb{R}^{2} .
$$

As a result $\int_{\varepsilon \square} \mathrm{d} \mu_{\varepsilon}=\varepsilon^{2} \int_{\square} \mathrm{d} \mu=\varepsilon^{2}$. Then the measure $\mu_{\varepsilon}$ weakly converges to the Lebesgue measure on $\mathbb{R}^{2}$, that is (see Zhikov [35])

$$
\lim _{\varepsilon \rightarrow 0} \int_{\mathbb{R}^{2}} \varphi \mathrm{d} \mu_{\varepsilon}=\int_{\mathbb{R}^{2}} \varphi \mathrm{d} x \quad \forall \varphi \in \mathrm{C}_{0}^{\infty}\left(\mathbb{R}^{2}\right) .
$$

Let $\mu^{S}$ be the $\square^{S}$-periodic measure in $\mathbb{R}^{1}$ defined as

$$
\mu^{S}=\sum_{j=1}^{L} \rho_{j} \delta_{M_{j}}
$$

where the nonnegative weights $\rho_{j}$ satisfy $\sum_{j=1}^{L} \rho_{j}=1$, and $\delta_{M_{j}}$ are the Dirac measures located at the vertices $M_{j}$. It is the easy to see that $\mu^{S}$ is the Radon measure, $\int_{\square S} \mathrm{~d} \mu^{S}=1$. So, we may then define the corresponding scaling measure by

$$
\mu_{\varepsilon}^{S}(B)=\varepsilon \mu^{S}\left(\varepsilon^{-1} B\right) \quad \text { for any Borel set } B \subset \mathbb{R}^{1} .
$$

Since $\int_{\varepsilon \square^{S}} \mathrm{~d} \mu_{\varepsilon}^{S}=\varepsilon$, it follows that $\mathrm{d} \mu_{\varepsilon}^{S}$ converges weakly to $\mathrm{d} \ell$ as $\varepsilon \rightarrow 0$, where by $\mathrm{d} \ell$ is denoted the linear Lebesgue measure in $\mathbb{R}^{1}$.

\section{Optimal CONTROL PROBlems ON $\varepsilon$-PeRIOdIC GRAPHS}

In order to formulate an optimal control problem on $\Omega_{\varepsilon}=\Omega \cap \varepsilon \mathcal{F}$, we introduce the Sobolev space $V\left(\Omega, \Gamma_{2}, \mathrm{~d} \mu\right)$, where $\mu$ is the non-negative $\square$-periodic Borel measure on $\mathbb{R}^{2}(2.2)$. Let us denote by $\mathrm{C}^{\infty}\left(\Omega, \Gamma_{2}\right)$ the class of smooth functions $\varphi \in \mathrm{C}^{\infty}(\bar{\Omega})$ such that $\left.\varphi\right|_{\Gamma_{2}}=0$. Here $\Gamma_{2}$ is the second "part" of the boundary $\partial \Omega=\Gamma_{1} \cup \Gamma_{2} . \mathrm{L}^{2}\left(\Omega, \mathrm{d} \mu_{\varepsilon}\right)$ and $\mathrm{L}^{2}\left(\Gamma_{1}, \mathrm{~d} \mu_{\varepsilon}^{S}\right)$ are defined as usual.

Definition 3.1. A function $y(x)$ belongs to $V\left(\Omega, \Gamma_{2}, \mathrm{~d} \mu\right)$ if there exists a vector $z \in\left(\mathrm{L}^{2}(\Omega, \mathrm{d} \mu)\right)^{2}$ and a sequence $\left\{y_{m} \in \mathrm{C}^{\infty}\left(\Omega, \Gamma_{2}\right)\right\}_{m \in N}$ such that

$$
\lim _{m \rightarrow \infty} \int_{\Omega}\left(y_{m}-y\right)^{2} \mathrm{~d} \mu=0, \quad \lim _{m \rightarrow \infty} \int_{\Omega}\left|\nabla y_{m}-z\right|^{2} \mathrm{~d} \mu=0
$$

In this case we say that $z$ is a gradient of $y$ and denote it as $\nabla y$, i.e. $z=\nabla y$.

Note that every function $y \in V\left(\Omega, \Gamma_{2}, \mathrm{~d} \mu\right)$ may have many gradients $z=\nabla y$. Moreover if we denote by $\Gamma(y)$ the set of gradients for a fixed function $y \in V\left(\Omega, \Gamma_{2}, \mathrm{~d} \mu\right)$ then this set has the following structure 
$\Gamma(y)=\nabla y+\Gamma(0)$ (see [8]), where $\nabla y \in \mathrm{L}^{2}(\Omega, \mathrm{d} \mu)^{2}$ is some fixed gradient and $\Gamma(0)$ is the set of gradients of zero. That is by definition, $g \in \Gamma(0)$ if there exists a sequence $\left\{\varphi_{m} \in \mathrm{C}^{\infty}(\bar{\Omega})\right\}$ such that

$$
\int_{\Omega} \varphi_{m}^{2} \mathrm{~d} \mu \longrightarrow 0 \text { as } m \rightarrow \infty, \quad \int_{\Omega}\left|\nabla \varphi_{m}-g\right|^{2} \mathrm{~d} \mu \longrightarrow 0 \text { as } m \rightarrow \infty .
$$

From this it follows immediately that $\Gamma(y)$ is a closed subspace of $\mathrm{L}^{2}(\Omega, \mathrm{d} \mu)^{2}$. Let us recall also that any gradient $\nabla y$ can be represented as a sum of two orthogonal terms: $\nabla y=\nabla^{t} y+g, g \in \Gamma(0), \nabla^{t} y \perp \Gamma(0)$. Hence (see [36]) the first term is also a gradient of $y$, and this gradient is minimal in the following sense:

$$
\int_{\Omega}\left|\nabla^{t} y\right|^{2} \mathrm{~d} \mu=\min _{\nabla y \in \Gamma(y)} \int_{\Omega}|\nabla y|^{2} \mathrm{~d} \mu .
$$

It is important to note that the space $\Gamma(0)$ admits a pointwise description. Namely, there is a $\mu$-measurable subspace $T(x) \subset \mathbb{R}^{2}$ such that $\Gamma(0)=\left\{g \in \mathrm{L}^{2}(\Omega, \mathrm{d} \mu)^{2}: g(x) \in T^{\perp}(x)\right\}$. So, the "minimal" gradient is determined by the tangential condition $\nabla y(x) \in T(x) \mu$-a.e.

The following result can be viewed as the necessary and sufficient conditions for the inclusion $y \in V\left(\Omega, \Gamma_{2}, \mathrm{~d} \mu\right)$, where the measure $\mu$ is defined in (2.3).

Proposition 3.2. Let $\mathcal{F}$ be $a \square$-periodic unbounded graph on $\mathbb{R}^{2}$, let $\mu$ be the $\square$-periodic Borel measure in $\mathbb{R}^{2}$ defined by (2.3) and let $y$ be any function of $V\left(\Omega, \Gamma_{2}, \mathrm{~d} \mu\right)$. Then:

(i) $\left.y\right|_{I_{i}} \in \mathrm{H}^{1}\left(I_{i}\right)$ for any edge $I_{i} \in \Omega \cap \mathcal{F}$, where $\mathrm{H}^{1}\left(I_{i}\right)$ is the one-dimensional Sobolev space on the segment $I_{i}$;

(ii) the values of $\left.y\right|_{I_{i}}$ coincide at the vertices of the graph.

This follows immediately from Lemma 3 in [8].

Notice, for $y \in V\left(\Omega, \Gamma_{2}, \mathrm{~d} \mu\right)$ its restriction on the set $\Omega \cap \mathcal{F}$ is continuous.

Let $\left\{A_{\varepsilon}(x): A_{\varepsilon}(\cdot) \in \mathrm{L}^{\infty}\left(\Omega, \mathbb{R}^{2 \times 2} ; \mathrm{d} \mu_{\varepsilon}\right)\right\}_{\varepsilon \in \mathbb{N}}$ be a family of matrices such that

$$
\alpha_{0}\|\xi\|^{2} \leq\left(A_{\varepsilon}(x) \xi, \xi\right) \leq \alpha_{0}^{-1}\|\xi\|^{2} \quad \text { for } \mu_{\varepsilon} \text {-a.e. } \quad x \in \Omega,
$$

where $\alpha_{0}>0$ is some constant which is independent of $\varepsilon$.

We define the optimal control problem on the $\varepsilon$-periodic graph-like domain $\Omega_{\varepsilon}=\Omega \cap \varepsilon \mathcal{F}$ as follows: seek a "boundary" control $h_{\varepsilon}^{0} \in \mathrm{L}^{2}\left(\Gamma_{1}, \mathrm{~d} \mu_{\varepsilon}^{S}\right)$, a "distributed" control $u_{\varepsilon}^{0} \in \mathrm{L}^{2}\left(\Omega, \mathrm{d} \mu_{\varepsilon}\right)$, and a corresponding state $y_{\varepsilon}^{0} \in V\left(\Omega, \Gamma_{2}, \mathrm{~d} \mu_{\varepsilon}\right)$ such that a cost functional

$$
I_{\varepsilon}\left(y_{\varepsilon}, u_{\varepsilon}, h_{\varepsilon}\right)=k_{1} \int_{\Omega}\left(y_{\varepsilon}-z_{d}\right)^{2} \mathrm{~d} \mu_{\varepsilon}+k_{2} \int_{\Omega} u_{\varepsilon}^{2} \mathrm{~d} \mu_{\varepsilon}+k_{3} \int_{\Gamma_{1}} h_{\varepsilon}^{2} \mathrm{~d} \mu_{\varepsilon}^{S}
$$

is minimized subject to the following constraints

$$
\begin{gathered}
y_{\varepsilon} \in V\left(\Omega, \Gamma_{2}, \mathrm{~d} \mu_{\varepsilon}\right), u_{\varepsilon} \in \mathrm{L}^{2}\left(\Omega, \mathrm{d} \mu_{\varepsilon}\right), h_{\varepsilon} \in \mathrm{L}^{2}\left(\Gamma_{1}, \mathrm{~d} \mu_{\varepsilon}^{S}\right), \\
\int_{\Omega}\left(A_{\varepsilon}(x) \nabla y_{\varepsilon}, \nabla \varphi\right) \mathrm{d} \mu_{\varepsilon}+\int_{\Omega} \alpha y_{\varepsilon} \varphi \mathrm{d} \mu_{\varepsilon}=\int_{\Omega} u_{\varepsilon} \varphi \mathrm{d} \mu_{\varepsilon}+\int_{\Gamma_{1}} h_{\varepsilon} \varphi \mathrm{d} \mu_{\varepsilon}^{S} \quad \forall \varphi \in C^{\infty}\left(\Omega, \Gamma_{2}\right),(\alpha>0), \\
\left|u_{\varepsilon}\right| \leq c_{u} \quad \mu_{\varepsilon} \text {-a.e. in } \Omega, \quad\left|h_{\varepsilon}\right| \leq c_{h} \quad \mu_{\varepsilon}^{S} \text {-a.e. on } \Gamma_{1} .
\end{gathered}
$$

Here $c_{u}$ and $c_{h}$ are some positive constants, $z_{d} \in \mathrm{C}^{0}(\bar{\Omega})$ is a given function, $\mu_{\varepsilon}$ and $\mu_{\varepsilon}^{S}$ are the "scaling" measures defined by (2.3) and (2.6), respectively, and $A_{\varepsilon}(x) \in \mathcal{L}\left(\mathbb{R}^{2}, \mathbb{R}^{2}\right)$ is a $\mu_{\varepsilon}$-measurable symmetric matrix satisfying the inequality (3.2), $k_{1}, k_{2}, k_{3}>0$ are given constants. Note that by the vector-function $\nabla y_{\varepsilon}$ in (3.5) 
we mean only that $\nabla y_{\varepsilon} \in \Gamma\left(y_{\varepsilon}\right)$ is some gradient of $y_{\varepsilon}$ in the sense of Definition 3.1 and $\nabla y_{\varepsilon}$ satisfies with $y_{\varepsilon}$ the integral identity (3.5).

We emphasize also that the notion "boundary" and "distributed" controls should be understood with respect to the corresponding measures and the integral identity (3.5). For example, the inclusion $h \in \mathrm{L}^{2}\left(\Gamma_{1}, \mathrm{~d} \mu_{\varepsilon}^{S}\right)$ implies that this function is uniquely defined by the respective set of values at the points $K_{\varepsilon}=\Gamma_{1} \cap\left(\cup_{n \in \mathbb{Z}}(\varepsilon \mathcal{M}+n \varepsilon)\right)$. Here $\mathcal{M}=\left\{M_{i}, i=1,2, \ldots, L\right\}$ is the set of all vertices on $\square$ which belong to $\square^{S}$. Note that by definition of the $\mu^{S}$ we have $\mu_{\varepsilon}^{S}\left(\Gamma_{1} \backslash K_{\varepsilon}\right)=0$.

First of all we show that for every $u_{\varepsilon} \in \mathrm{L}^{2}\left(\Omega, \mathrm{d} \mu_{\varepsilon}\right), h_{\varepsilon} \in \mathrm{L}^{2}\left(\Gamma_{1}, \mathrm{~d} \mu_{\varepsilon}^{S}\right)$, and $\varepsilon \in E$ there exists a unique pair $\left(y_{\varepsilon}, \nabla y_{\varepsilon}\right)$ that satisfies identity (3.5).

Lemma 3.3. Under the standing assumption on the measures $\mu$ and $\mu^{S}$ and the matrix $A_{\varepsilon}$ there exists a unique $y_{\varepsilon} \in V\left(\Omega, \Gamma_{2}, \mathrm{~d} \mu_{\varepsilon}\right)$ and a unique gradient $\nabla y_{\varepsilon} \in \Gamma\left(y_{\varepsilon}\right)$ satisfying (3.5).

Proof. Since the set $\mathrm{C}^{\infty}\left(\Omega, \Gamma_{2}\right)$ is dense in the class $V\left(\Omega, \Gamma_{2}, \mathrm{~d} \mu_{\varepsilon}\right)$, the left-hand side of (3.5) induces a new scalar product on $\mathrm{L}^{2}\left(\Omega, \mathrm{d} \mu_{\varepsilon}\right) \times \mathrm{L}^{2}\left(\Omega, \mathrm{d} \mu_{\varepsilon}\right)^{2}$, and the corresponding norm is equivalent to the usual norm in this space. Thus the existence and uniqueness of the solution regarded as the pair $\left(y_{\varepsilon}, \nabla y_{\varepsilon}\right)$ is an easy consequence of the Lax-Milgram lemma. However, the uniqueness is twofold here: there exists a unique function $y_{\varepsilon}$ of the Sobolev space $V\left(\Omega, \Gamma_{2}, \mathrm{~d} \mu_{\varepsilon}\right)$ such that only one of its gradients satisfies the identity (3.5). The uniqueness and existence of such gradient was proved in [35]. It is interesting to note that the gradient $\nabla y_{\varepsilon}$ in this identity is defined only by matrix $A_{\varepsilon}$ alone and it is not related to the equation itself (for details we refer to Zhikov [35]).

Remark 3.4. It is easy to see that the solution of (3.5) satisfies the following estimate

$$
\left\|y_{\varepsilon}\right\|_{\mathrm{L}^{2}\left(\Omega, \mathrm{d} \mu_{\varepsilon}\right)}+\left\|\nabla y_{\varepsilon}\right\|_{\left(\mathrm{L}^{2}\left(\Omega, \mathrm{d} \mu_{\varepsilon}\right)\right)^{2}} \leq \alpha^{-1}\left(\left\|u_{\varepsilon}\right\|_{\mathrm{L}^{2}\left(\Omega, \mathrm{d} \mu_{\varepsilon}\right)}+\left\|h_{\varepsilon}\right\|_{\mathrm{L}^{2}\left(\Gamma_{1}, \mathrm{~d} \mu_{\varepsilon}^{S}\right)}\right)
$$

for every $\varepsilon \in E, u_{\varepsilon} \in \mathrm{L}^{2}\left(\Omega, \mathrm{d} \mu_{\varepsilon}\right)$ and $h_{\varepsilon} \in \mathrm{L}^{2}\left(\Gamma_{1}, \mathrm{~d} \mu_{\varepsilon}^{S}\right)$, where the constant $\alpha$ is independent of $\varepsilon$. Indeed, if we take $\varphi=y_{\varepsilon}$ as test function in (3.5) and use the Young's inequality, we immediately obtain the required relation (3.7).

Definition 3.5. The triplet $(y, u, h) \in \mathbb{Z}_{\varepsilon}\left(\Omega, \Gamma_{1}\right)$ is called admissible if $(y, u, h)$ satisfies the restrictions (3.4)(3.6). We denote by $\Xi_{\varepsilon}$ the set of all admissible triplets for the optimal control problem (3.3)-(3.6).

Remark 3.6. In view of (3.6) and estimate (3.7) we see that the sequence of sets $\left\{\Xi_{\varepsilon}\right\}_{\varepsilon \in E}$ is uniformly bounded in the following sense: there is a constant $C>0$ such that

$$
\sup _{\varepsilon \in E} \sup _{\left(y_{\varepsilon}, u_{\varepsilon}, h_{\varepsilon}\right) \in \Xi_{\varepsilon}}\left[\left\|y_{\varepsilon}\right\|_{\mathrm{L}^{2}\left(\Omega, \mathrm{d} \mu_{\varepsilon}\right)}+\left\|u_{\varepsilon}\right\|_{\mathrm{L}^{2}\left(\Omega, \mathrm{d} \mu_{\varepsilon}\right)}+\left\|h_{\varepsilon}\right\|_{\mathrm{L}^{2}\left(\Gamma_{1}, \mathrm{~d} \mu_{\varepsilon}^{S}\right)}+\left\|\nabla y_{\varepsilon}\right\|_{\left(\mathrm{L}^{2}\left(\Omega, \mathrm{d} \mu_{\varepsilon}\right)\right)^{2}}\right] \leq C .
$$

Moreover, each of the sets $\Xi_{\varepsilon}$ is convex and closed with respect to the weak convergence in the space $\mathrm{L}^{2}\left(\Omega, \mathrm{d} \mu_{\varepsilon}\right) \times$ $\mathrm{L}^{2}\left(\Omega, \mathrm{d} \mu_{\varepsilon}\right) \times \mathrm{L}^{2}\left(\Gamma_{1}, \mathrm{~d} \mu_{\varepsilon}^{S}\right)$. Indeed, let $\left\{u_{n}\right\}_{n \in \mathbb{N}} \subset \mathrm{L}^{2}\left(\Omega, \mathrm{d} \mu_{\varepsilon}\right)$ and $\left\{h_{n}\right\}_{n \in \mathbb{N}} \subset \mathrm{L}^{2}\left(\Gamma_{1}, \mathrm{~d} \mu_{\varepsilon}^{S}\right)$ be any bounded sequences such that

$$
u_{n} \rightarrow u \text { weakly in } \mathrm{L}^{2}\left(\Omega, \mathrm{d} \mu_{\varepsilon}\right), \quad h_{n} \rightarrow h \text { weakly in } \mathrm{L}^{2}\left(\Gamma_{1}, \mathrm{~d} \mu_{\varepsilon}^{S}\right) .
$$

We define the sequence $\left\{y_{n} \in \mathrm{H}^{1}\left(\Omega, \mathrm{d} \mu_{\varepsilon}\right)\right\}$ as the corresponding solutions of the problem (3.5) under $u=u_{n}$ and $h=h_{n}$. Then by inequality (3.7) there exists a constant $C>0$ such that

$$
\left\|y_{n}\right\|_{\mathrm{L}^{2}\left(\Omega, \mathrm{d} \mu_{\varepsilon}\right)} \leq C, \quad\left\|\nabla y_{n}\right\|_{\left(\mathrm{L}^{2}\left(\Omega, \mathrm{d} \mu_{\varepsilon}\right)\right)^{2}} \leq C,
$$

where for every $n \in \mathbb{N} \nabla y_{n}$ is a unique gradient of $y_{n}$ satisfying the integral identity (3.5) under suitable $u_{n}$ and $h_{n}$.

Hence we may always assume that there is a function $y \in \mathrm{L}^{2}\left(\Omega, \mathrm{d} \mu_{\varepsilon}\right)$ and a vector $p \in \mathrm{L}^{2}\left(\Omega, \mathrm{d} \mu_{\varepsilon}\right)^{2}$ such that

$$
y_{n} \rightarrow y \text { weakly in } \mathrm{L}^{2}\left(\Omega, \mathrm{d} \mu_{\varepsilon}\right), \quad \nabla y_{n} \rightarrow p \text { weakly in } \mathrm{L}^{2}\left(\Omega, \mathrm{d} \mu_{\varepsilon}\right)^{2} .
$$


Therefore, using (3.9)-(3.10) we can pass to the limit as $n \rightarrow \infty$ in

$$
\int_{\Omega}\left[\left(A_{\varepsilon} \nabla y_{n}, \nabla \varphi\right)+\alpha y_{n} \varphi\right] \mathrm{d} \mu_{\varepsilon}=\int_{\Omega} u_{n} \varphi \mathrm{d} \mu_{\varepsilon}+\int_{\Omega} h_{n} \varphi \mathrm{d} \mu_{\varepsilon}^{S}
$$

and obtain

$$
\int_{\Omega}\left[\left(A_{\varepsilon} p, \nabla \varphi\right)+\alpha y \varphi\right] \mathrm{d} \mu_{\varepsilon}=\int_{\Omega} u \varphi \mathrm{d} \mu_{\varepsilon}+\int_{\Gamma_{1}} h \varphi \mathrm{d} \mu_{\varepsilon}^{S} \quad \forall \varphi \in \mathrm{C}^{\infty}\left(\Omega, \Gamma_{2}\right) .
$$

This proves that $p \in \Gamma_{\varepsilon}(y)$, i.e. $p=\nabla y$ is some gradient of the function $y \in \mathrm{H}^{1}\left(\Omega, \mathrm{d} \mu_{\varepsilon}\right)$. As a result, we have obtained that for every $\varepsilon \in E$ the set $\Xi_{\varepsilon}$ is sequentially closed with respect to the weak convergence.

We thus obtain the following:

Lemma 3.7. For every $\varepsilon \in E$ the optimal control problem (3.3)-(3.6) has a unique solution, i.e. there exists a unique triplet $\left(y_{\varepsilon}^{0}, u_{\varepsilon}^{0}, h_{\varepsilon}^{0}\right) \in \Xi_{\varepsilon}$ such that

$$
I_{\varepsilon}\left(y_{\varepsilon}^{0}, u_{\varepsilon}^{0}, h_{\varepsilon}^{0}\right)=\inf _{\left(y_{\varepsilon}, u_{\varepsilon}, h_{\varepsilon}\right) \in \Xi_{\varepsilon}} I_{\varepsilon}\left(y_{\varepsilon}, u_{\varepsilon}, h_{\varepsilon}\right) .
$$

Note that the uniqueness of this solution is a consequence of the convexity property for $\Xi_{\varepsilon}$ and strictly convexity of the cost functional $I_{\varepsilon}$.

At was mentioned in Introduction, the main question of our paper is devoted to the study of the asymptotic behavior of the optimal control problem (3.3)-(3.6) as $\varepsilon \rightarrow 0$. To this end we represent this problem for $\varepsilon \in E$ in the form of a sequence of constrained minimization problems

$$
\left\{\left\langle\inf _{\left(y_{\varepsilon}, u_{\varepsilon}, h_{\varepsilon}\right) \in \Xi_{\varepsilon}} I_{\varepsilon}\left(y_{\varepsilon}, u_{\varepsilon}, h_{\varepsilon}\right)\right\rangle ; \varepsilon \rightarrow 0\right\}
$$

where the cost functional $I_{\varepsilon}: \Xi_{\varepsilon} \rightarrow \mathbb{R}$ and the sets of admissible triplets are defined in (3.3) and (3.4)-(3.6), respectively. Then the definition of an appropriate homogenized optimal control problem to the family (3.3)(3.6) as $\varepsilon \rightarrow 0$ can be reduced to the analysis of the limit properties of the sequences (3.11). This will be done through the concept of variational convergence of constrained minimization problems $[17,19]$.

Definition 3.8. We say that the optimal control problem (3.3)-(3.6) has a homogenized limit problem as $\varepsilon$ tends to zero, if:

(i) for the sequence of the corresponding constrained minimization problems (3.11) there exists a variational limit as $\varepsilon \rightarrow 0$, i.e.

$$
\left\langle\inf _{\left(y_{\varepsilon}, u_{\varepsilon}, h_{\varepsilon}\right) \in \Xi_{\varepsilon}} I_{\varepsilon}\left(y_{\varepsilon}, u_{\varepsilon}, h_{\varepsilon}\right)\right\rangle \longrightarrow\left\langle\inf _{(y, u, h) \in \Xi_{0}} I_{0}(y, u, h)\right\rangle \quad \text { as } \epsilon \rightarrow 0 ;
$$

(ii) the minimization problem (3.12) can be recovered in the form of some optimal control problem.

The sense in which this convergence holds is going to be developed in Definition 5.5. Before we embark on the development of the necessary formalism for such convergence, we note that it will have to preserve the main variational property: both the optimal triplet and minimal value of the cost functional for the problem (3.11) converge to the corresponding characteristics of a limit minimization problem as $\varepsilon$ tends to zero.

\section{Convergence in SPACES ON $\varepsilon$-PERIODiC GRAPHS}

We begin this section with brief description of the main results concerning the convergence in the variable $\mathrm{L}^{2}$-spaces following the papers $[34,35]$. We also introduce some additional spaces associated with boundary optimal control problems on $\varepsilon$-periodic graphs. 
Assuming the boundedness of the sequence $\left\{h_{\varepsilon} \in \mathrm{L}^{2}\left(\Gamma_{1}, \mathrm{~d} \mu_{\varepsilon}^{S}\right)\right\}$, i.e., $\limsup _{\varepsilon \rightarrow 0} \int_{\Gamma_{1}} h_{\varepsilon}^{2} \mathrm{~d} \mu_{\varepsilon}^{S}<\infty$, we say that:

(1) $h_{\varepsilon} \rightarrow h \in \mathrm{L}^{2}\left(\Gamma_{1}\right)$ weakly in $\mathrm{L}^{2}\left(\Gamma_{1}, \mathrm{~d} \mu_{\varepsilon}^{S}\right)$, if $\lim _{\varepsilon \rightarrow 0} \int_{\Gamma_{1}} \varphi h_{\varepsilon} \mathrm{d} \mu_{\varepsilon}^{S}=\int_{\Gamma_{1}} \varphi h \mathrm{~d} l$ for every $\varphi \in \mathrm{C}_{0}^{\infty}\left(\Gamma_{1}\right)$;

(2) $h_{\varepsilon} \rightarrow h \in \mathrm{L}^{2}\left(\Gamma_{1}\right)$ strongly in $\mathrm{L}^{2}\left(\Gamma_{1}, \mathrm{~d} \mu_{\varepsilon}^{S}\right)$, if $\lim _{\varepsilon \rightarrow 0} \int_{\Gamma_{1}} h_{\varepsilon} p_{\varepsilon} \mathrm{d} \mu_{\varepsilon}^{S}=\int_{\Gamma_{1}} h p \mathrm{~d} l$ for any $\left\{p_{\varepsilon} \in \mathrm{L}^{2}\left(\Gamma_{1}, \mathrm{~d} \mu_{\varepsilon}^{S}\right)\right\}$ such that $p_{\varepsilon} \rightarrow p$ in $\mathrm{L}^{2}\left(\Gamma_{1}, \mathrm{~d} \mu_{\varepsilon}^{S}\right)$.

We now give the main properties of convergence in variable space following Zhikov [35].

Proposition 4.1. (a) Compactness criterium: Every bounded sequence $\left\{h_{\varepsilon} \in \mathrm{L}^{2}\left(\Gamma_{1}, \mathrm{~d} \mu_{\varepsilon}^{S}\right)\right\}$ is relatively compact with respect to the weak convergence in $\mathrm{L}^{2}\left(\Gamma_{1}, \mathrm{~d} \mu_{\varepsilon}^{S}\right)$.

(b) Property of lower semicontinuity: If $h_{\varepsilon} \rightarrow h$, then $\liminf _{\varepsilon \rightarrow 0} \int_{\Gamma_{1}} h_{\varepsilon}^{2} \mathrm{~d} \mu_{\varepsilon}^{S} \geq \int_{\Gamma_{1}} h^{2} \mathrm{~d} l$.

(c) Criterium of strong convergence: The weak convergence of $h_{\varepsilon} \rightarrow h$ and the relation

$$
\lim _{\varepsilon \rightarrow 0} \int_{\Gamma_{1}} h_{\varepsilon}^{2} \mathrm{~d} \mu_{\varepsilon}^{S}=\int_{\Gamma_{1}} h^{2} \mathrm{~d} l
$$

imply the strong convergence of $h_{\varepsilon} \rightarrow h$ in $\mathrm{L}^{2}\left(\Gamma_{1}, \mathrm{~d} \mu_{\varepsilon}^{S}\right)$.

Now we define two-scale convergence in $\mathrm{L}^{2}\left(\Gamma_{1}, \mathrm{~d} \mu_{\varepsilon}^{S}\right)$. Note that the main results concerning the extension of the well known method of two-scale convergence was independently obtained in [35] and [3]. We give the definition and main properties of two-scale limits with respect to the 'scaling' measure $\mu_{\varepsilon}^{S}$. This measure, as follows from Section 2, is singular with respect to the $\mu_{\varepsilon}$ and associated with a "boundary condition" on the set $\partial\left(\Omega \cap \mathcal{F}_{\varepsilon}\right)$. Let $\left\{h_{\varepsilon} \in \mathrm{L}^{2}\left(\Gamma_{1}, \mathrm{~d} \mu_{\varepsilon}^{S}\right)\right\}$ be a bounded sequence in $\mathrm{L}^{2}\left(\Gamma_{1}, \mathrm{~d} \mu_{\varepsilon}^{S}\right)$. We say that this sequence weakly two-scale converges to a function $h \in \mathrm{L}^{2}\left(\Gamma_{1}, \square^{S}\right)$, where $h=h(l, \xi), \mathrm{L}^{2}\left(\Gamma_{1}, \square^{S}\right)=\mathrm{L}^{2}\left(\Gamma_{1} \times \square^{S}, \mathrm{~d} l \times \mathrm{d} \mu^{S}\right)$ if

$$
\lim _{\varepsilon \rightarrow 0} \int_{\Gamma_{1}} \Psi\left(l, \varepsilon^{-1} l\right) h_{\varepsilon}(l) \mathrm{d} \mu_{\varepsilon}^{S}=\int_{\Gamma_{1}} \int_{\square} \Psi(l, \xi) h(l, \xi) \mathrm{d} l \mathrm{~d} \mu^{S}(\xi)
$$

for every $\Psi(l, \xi)=a(l) \eta(\xi)$ such that $a \in \mathrm{C}^{0}\left(\bar{\Gamma}_{1}\right)$ and $\eta \in \mathrm{L}_{\text {per }}^{2}\left(\square^{S}\right)$ (in this case, we write $h_{\varepsilon} \stackrel{2}{\rightarrow} h$ ). Note that at the heart of this concept there is the following mean value property of periodic functions.

Proposition 4.2. Assume that $\eta$ is any $\mu^{S}$-measurable $\square^{S}$-periodic function on $\mathbb{R}^{1}$. Then for any $a \in \mathrm{C}_{0}^{\infty}\left(\mathbb{R}^{1}\right)$ the following equality holds true:

$$
\lim _{\varepsilon \rightarrow 0} \int_{\Gamma_{1}} a(l) \eta\left(\varepsilon^{-1} l\right) \mathrm{d} \mu_{\varepsilon}^{S}=\langle\eta\rangle^{S} \cdot \int_{\Gamma_{1}} a(l) \mathrm{d} l,
$$

where $\langle\eta\rangle^{S}=\int_{\square^{S}} \eta(\xi) \mathrm{d} \mu^{S}(\xi)$ is the mean value of $\eta$ on the cell $\square^{S}$.

We say that a sequence $\left\{h_{\varepsilon} \in \mathrm{L}^{2}\left(\Gamma_{1}, \mathrm{~d} \mu_{\varepsilon}^{S}\right)\right\}$ strongly two-scale converges to a function $h=h(l, \xi) \in$ $\mathrm{L}^{2}\left(\Gamma_{1}, \square^{S}\right)$ if

$$
\lim _{\varepsilon \rightarrow 0} \int_{\Gamma_{1}} h_{\varepsilon}(x) v_{\varepsilon}(x) \mathrm{d} \mu_{\varepsilon}^{S}=\int_{\Gamma_{1}} \int_{\square^{S}} h(l, \xi) v(l, \xi) \mathrm{d} x \mathrm{~d} \mu^{S}(\xi)
$$

for any $v_{\varepsilon} \stackrel{2}{\rightarrow} v(l, \xi)$. In this case, we write $h_{\varepsilon} \stackrel{2}{\rightarrow} h(l, \xi)$. 
Let us list some general properties of two-scale-convergence which we apply below.

(i) If a sequence is bounded in $\mathrm{L}^{2}\left(\Gamma_{1}, \mathrm{~d} \mu_{\varepsilon}^{S}\right)$, then it contains a weakly two-scale convergent subsequence.

(ii) If $h_{\varepsilon} \stackrel{2}{\rightarrow} h \in \mathrm{L}^{2}\left(\Gamma_{1}, \square^{S}\right)$, then $\liminf _{\varepsilon \rightarrow 0} \int_{\Gamma_{1}} h_{\varepsilon}^{2} \mathrm{~d} \mu_{\varepsilon}^{S} \geq \int_{\Gamma_{1}} \int_{\square^{S}} h^{2}(l, \xi) \mathrm{d} l \mathrm{~d} \mu^{S}(\xi)$.

(iii) If $h_{\varepsilon} \stackrel{2}{\rightarrow} h(l, \xi)$, then $h_{\varepsilon} \rightarrow \int_{\square S} h(l, \xi) \mathrm{d} \mu^{S}(\xi)$.

(iv) $h_{\varepsilon} \stackrel{2}{\rightarrow} h(l, \xi) \Leftrightarrow h_{\varepsilon} \stackrel{2}{\rightarrow} h$ and $\lim _{\varepsilon \rightarrow 0} \int_{\Gamma_{1}} h_{\varepsilon}^{2} \mathrm{~d} \mu_{\varepsilon}^{S}=\int_{\Gamma_{1}} \int_{\square^{S}} h^{2}(l, \xi) \mathrm{d} l \mathrm{~d} \mu^{S}(\xi)$.

(v) If $h_{\varepsilon} \rightarrow h(l)$ strongly in $\mathrm{L}^{2}\left(\Gamma_{1}, \square^{S}\right)$, and $\lim _{\varepsilon \rightarrow 0} \int_{\Gamma_{1}} h_{\varepsilon}^{2} \mathrm{~d} \mu_{\varepsilon}^{S}=\int_{\Gamma_{1}} h^{2}(l) \mathrm{d} l$, then $h_{\varepsilon} \stackrel{2}{\rightarrow} h(l)$, that is, the two-scale limit is independent of the second variable $\xi$. Note that the similar notions and results can be applied to the space $\mathrm{L}^{2}\left(\Omega, \mathrm{d} \mu_{\varepsilon}\right)$.

Now we define the sets of so-called potential and solenoidal vectors on the cell of periodicity $\square$ (or the torus). Let $\mathrm{C}_{\text {per }}^{\infty}=\mathrm{C}_{\text {per }}^{\infty}(\square)$ be the space of infinitely differentiable periodic functions, let $\mathrm{L}_{\text {per }}^{2}(\square, \mathrm{d} \mu)^{2}$ be the space of $\mu$-measurable periodic functions $f=\left[f_{1}, f_{2}\right]$ such that $\int_{\Omega}\left(f_{1}^{2}(x)+f_{2}^{2}(x)\right) \mathrm{d} \mu<\infty$. We say that a vector-function $g$ belongs to the space $V_{\text {pot }}$ of potential vectors if there exists a sequence $\left\{\varphi_{m} \in \mathrm{C}_{\text {per }}^{\infty}\right\}$ such that

$$
\nabla \varphi_{m} \rightarrow g \text { in } \mathrm{L}_{\mathrm{per}}^{2}(\square, \mathrm{d} \mu)^{2}, \quad \text { i.e. } \quad \int_{\square}\left|\nabla \varphi_{m}-g\right|^{2} \mathrm{~d} \mu \rightarrow 0 \text { as } m \rightarrow \infty \text {. }
$$

We also say that a vector-function $b$ belongs to the space $V_{\text {sol }}$ of solenoidal vectors if $b$ is orthogonal to all potential vectors, i.e. $\int_{\square}(b, g)_{\mathbb{R}^{2}} \mathrm{~d} \mu=0$ for every $g \in V_{\text {pot }}$. In view of this we always have the decomposition

$$
\mathrm{L}^{2}(\square, \mathrm{d} \mu)^{2}=V_{\mathrm{pot}} \oplus V_{\mathrm{sol}}
$$

Let us denote by $\mathrm{L}^{2}(\Omega, \square)$ the following space $\mathrm{L}^{2}(\Omega \times \square, \mathrm{d} x \times \mathrm{d} \mu)$, i.e. $y(x, z) \in \mathrm{L}^{2}(\Omega ; \square)$ if $y$ is $\mathrm{d} x \times \mathrm{d} \mu$ measurable on $\Omega \times \square$ and $\iint_{\Omega} y^{2}(x, z) \mathrm{d} x \mathrm{~d} \mu(z)<\infty$. Then from (4.2) we immediately have that

$$
\mathrm{L}^{2}(\Omega, \square)^{2} \equiv \mathrm{L}^{2}\left(\Omega, \mathrm{L}^{2}(\square)^{2}\right)=\mathrm{L}^{2}\left(\Omega, V_{\text {pot }}\right) \oplus \mathrm{L}^{2}\left(\Omega, V_{\text {sol }}\right) .
$$

Here by $\mathrm{L}^{2}\left(\Omega, V_{\text {pot }}\right)$ and $\mathrm{L}^{2}\left(\Omega, V_{\text {sol }}\right)$ we may understand the following spaces:

(i) $\mathrm{L}^{2}\left(\Omega, V_{\text {pot }}\right)$ is the closure in $\mathrm{L}^{2}(\Omega, \square)$ of the linear span of the vectors $f(x) \nabla_{z} \varphi(z)$, where $f \in \mathrm{C}_{0}^{\infty}(\Omega)$ and $\varphi \in \mathrm{C}_{\mathrm{per}}^{\infty}$

(ii) $\mathrm{L}^{2}\left(\Omega, V_{\text {sol }}\right)$ is the closure in $\mathrm{L}^{2}(\Omega, \square)$ of the linear span of the vectors $f(x) b(z)$, where $f \in \mathrm{C}_{0}^{\infty}(\Omega)$ and $b \in V_{\text {sol }}$.

Let us recall some properties of the $\square$-periodic Borel measure $\mu$ introduced above (see [35]).

Theorem 4.3. Let $\mu$ be the non-negative periodic Borel measure in $\mathbb{R}^{2}$ which is defined in (2.3). Then $\mu$ is non-degenerate and ergodic, i.e.

(i) Non-degenerateness property: For every vector $\eta \in \mathbb{R}^{2}$ there exists a vector $b \in V_{\text {pot }}$ such that $\int b(z) \mathrm{d} \mu=\eta$.

(ii) Ergodicity property: Every function $y \in \mathrm{H}^{1}(\square, \mathrm{d} \mu)$ with gradient zero is constant $\mu$-everywhere.

Remark 4.4. The validity of this theorem can be illustrated by the following observation. In view of (2.3) we have $\mu(\Omega \backslash \mathcal{F})=0$. Therefore any functions taking the same values on the graph $\Omega \cap \mathcal{F}$ coincide as elements 
of $\mathrm{L}^{2}(\Omega, \mathrm{d} \mu)$. Thus every element of the space $\mathrm{H}^{1}(\Omega, \mathrm{d} \mu)$ is uniquely defined by the respective element of the space $\cup \mathrm{H}^{1}\left(I_{j}\right)$, where $\left\{I_{j}\right\}$ is the set of all edges on the graph $\Omega \cap \mathcal{F}$.

Let $\left\{\left(y_{\varepsilon}, u_{\varepsilon}, h_{\varepsilon}\right) \in \Xi_{\varepsilon}\right\}_{\varepsilon \in E}$ be any sequence of admissible triplets. For every fixed $\varepsilon$ each of these triplets $\left(y_{\varepsilon}, u_{\varepsilon}, h_{\varepsilon}\right)$ belongs to the corresponding functional space $\mathbb{Z}_{\varepsilon}\left(\Omega, \Gamma_{1}\right) \equiv V\left(\Omega, \Gamma_{2}, \mathrm{~d} \mu_{\varepsilon}\right) \times \mathrm{L}^{2}\left(\Omega, \mathrm{d} \mu_{\varepsilon}\right) \times \mathrm{L}^{2}\left(\Gamma_{1}, \mathrm{~d} \mu_{\varepsilon}^{S}\right)$ depending on the small parameter $\varepsilon$. So, we focus our attention further on the convergence formalism in this variable space.

Definition 4.5. We say that the sequence of triplets $\left\{\left(y_{\varepsilon}, u_{\varepsilon}, h_{\varepsilon}\right)\right\}_{\varepsilon \in E}$ is weakly convergent in the variable space $\mathbb{Z}_{\varepsilon}\left(\Omega, \Gamma_{1}\right)$, or shortly, is $w$-convergent, if there are functions

$$
y \in \mathrm{H}^{1}(\Omega), u \in \mathrm{L}^{2}(\Omega), h \in \mathrm{L}^{2}\left(\Gamma_{1}\right), p \in \mathrm{L}^{2}(\Omega, \square)^{2},
$$

and there exists a sequence of gradients $\left\{\nabla y_{\varepsilon} \in \mathrm{L}^{2}\left(\Omega, \mathrm{d} \mu_{\varepsilon}\right)^{2}\right\}_{\varepsilon \in E}$ such that

$$
\begin{gathered}
u_{\varepsilon} \rightarrow u \text { in } \mathrm{L}^{2}\left(\Omega, \mathrm{d} \mu_{\varepsilon}\right), \quad h_{\varepsilon} \rightarrow h \text { in } \mathrm{L}^{2}\left(\Gamma_{1}, \mathrm{~d} \mu_{\varepsilon}^{S}\right), \\
y_{\varepsilon} \stackrel{2}{\rightarrow} y(x) \text { in } \mathrm{L}^{2}\left(\Omega, \mathrm{d} \mu_{\varepsilon}\right), \quad \nabla y_{\varepsilon} \stackrel{2}{\rightarrow} p(x, z) \text { in } \mathrm{L}^{2}\left(\Omega, \mathrm{d} \mu_{\varepsilon}\right)^{2}, \quad p(x, y)-\nabla y(x) \in \mathrm{L}^{2}\left(\Omega, V_{\text {pot }}\right) .
\end{gathered}
$$

Remark 4.6. Note that in Definition 4.5 a two-scale limit $y$ has to be independent of the second variable $z$. Moreover, as follows from (4.5) we do not conjecture that for the family of $\mathrm{H}^{1}$-functions $\left\{y_{\varepsilon} \in V\left(\Omega, \Gamma_{2}, \mathrm{~d} \mu_{\varepsilon}\right)\right\}$ there exists a sequence of gradients $\left\{\nabla y_{\varepsilon}\right\}_{\varepsilon \in E}$ such that $\nabla y_{\varepsilon} \rightarrow \nabla y \mu$-weakly or two-scale weakly.

As we will see later the conditions (4.4), (4.5) will be sufficient in order to identify the limit optimal control problem on the graph. In the following theorem we establish sufficient conditions of the relative $w$-compactness of uniformly bounded sequences.

Theorem 4.7. Let $\left\{\left(y_{\varepsilon}, u_{\varepsilon}, h_{\varepsilon}\right) \in \mathbb{Z}_{\varepsilon}\left(\Omega, \Gamma_{1}\right)\right\}_{\varepsilon \in E}$ be a sequence for which the following conditions hold:

(i) there exists a constant $C>0$ such that

$$
\sup _{\varepsilon}\left\{\left\|y_{\varepsilon}\right\|_{\mathrm{L}^{2}\left(\Omega, \mathrm{d} \mu_{\varepsilon}\right)},\left\|u_{\varepsilon}\right\|_{\mathrm{L}^{2}\left(\Omega, \mathrm{d} \mu_{\varepsilon}\right)},\left\|h_{\varepsilon}\right\|_{\mathrm{L}^{2}\left(\Omega, \mathrm{d} \mu_{\varepsilon}^{S}\right)}\right\} \leq C
$$

(ii) there exists a bounded sequence of gradients $\left\{\nabla y_{\varepsilon} \in \mathrm{L}^{2}\left(\Omega, \mathrm{d} \mu_{\varepsilon}\right)^{2}\right\}$, i.e. $\limsup _{\varepsilon \rightarrow 0} \int_{\Omega}\left|\nabla y_{\varepsilon}\right|^{2} \mathrm{~d} \mu_{\varepsilon}<+\infty$.

Then the sequence $\left\{\left(y_{\varepsilon}, u_{\varepsilon}, h_{\varepsilon}\right)\right\}_{\varepsilon \in E}$ is relatively compact with respect to the w-convergence.

Proof. First of all we note that in view of the properties of the weak (two-scale) convergence in the variable spaces, it may be supposed without loss of generality that

$$
\left.\begin{array}{llll}
y_{\varepsilon} & \stackrel{2}{\rightarrow} & y(x, z) \in \mathrm{L}^{2}(\Omega, \square), & h_{\varepsilon} \rightarrow h(l)=\int_{\square} h(l, \xi) \mathrm{d} \mu^{S}(\xi) \quad \text { in } \mathrm{L}^{2}\left(\Gamma_{1}, \mathrm{~d} \mu_{\varepsilon}^{S}\right), \\
\nabla y_{\varepsilon} & \stackrel{2}{\rightarrow} p(x, z) \in \mathrm{L}^{2}(\Omega, \square), & u_{\varepsilon} \rightarrow u(x)=\int_{\square} u(x, z) \mathrm{d} \mu(z) \quad \text { in } \mathrm{L}^{2}\left(\Omega, \mathrm{d} \mu_{\varepsilon}\right) .
\end{array}\right\}
$$

Let $b \in \mathrm{L}^{2}(\square, \mathrm{d} \mu)^{2}$ be a vector-valued function for which there is an element $a \in \mathrm{L}^{2}(\square, \mathrm{d} \mu)$ such that

$$
-\int_{\square} a(z) \varphi(z) \mathrm{d} \mu=\int_{\square}(b(z), \nabla \varphi(z)) \mathrm{d} \mu \quad \forall \varphi \in \mathrm{C}_{\text {per }}^{\infty}(\square) .
$$

Then taking $\varphi \in \mathrm{C}_{0}^{\infty}(\Omega)$ as a test function and using the equality $\nabla\left(\varphi y_{\varepsilon}\right)=\varphi \nabla y_{\varepsilon}+y_{\varepsilon} \nabla \varphi$, we have

$$
\varepsilon \int_{\Omega}\left(\nabla y_{\varepsilon}(x) \varphi(x), b\left(\varepsilon^{-1} x\right)\right) \mathrm{d} \mu_{\varepsilon}=\varepsilon \int_{\Omega}\left(\nabla\left(\varphi y_{\varepsilon}\right), b\left(\varepsilon^{-1} x\right)\right) \mathrm{d} \mu_{\varepsilon}-\varepsilon \int_{\Omega} y_{\varepsilon}(x)\left(\nabla \varphi(x), b\left(\varepsilon^{-1} x\right)\right) \mathrm{d} \mu_{\varepsilon},
$$


and

$$
-\int_{\Omega} a\left(\varepsilon^{-1} x\right) \varphi(x) \mathrm{d} \mu_{\varepsilon}=\varepsilon \int_{\Omega}\left(b\left(\varepsilon^{-1} x\right), \nabla \varphi\right) \mathrm{d} \mu_{\varepsilon} .
$$

Therefore, applying (4.10) to (4.9) we obtain

$$
\varepsilon \int_{\Omega} \varphi(x)\left(\nabla y_{\varepsilon}(x), b\left(\varepsilon^{-1} x\right)\right) \mathrm{d} \mu_{\varepsilon}=-\int_{\Omega} a\left(\varepsilon^{-1} x\right) y_{\varepsilon}(x) \varphi(x) \mathrm{d} \mu_{\varepsilon}-\varepsilon \int_{\Omega} y_{\varepsilon}(x)\left(\nabla \varphi(x), b\left(\varepsilon^{-1} x\right)\right) \mathrm{d} \mu_{\varepsilon} .
$$

Taking (4.7) and supposition (ii) into account one gets

$$
\lim _{\varepsilon \rightarrow 0} \varepsilon \cdot\left\|\nabla y_{\varepsilon}\right\|_{\left(\mathrm{L}^{2}\left(\Omega, \mathrm{d} \mu_{\varepsilon}\right)\right)^{2}}=0, \quad \lim _{\varepsilon \rightarrow 0} \varepsilon \int_{\Omega} \varphi(x)\left(\nabla y_{\varepsilon}(x), b\left(\varepsilon^{-1} x\right)\right) \mathrm{d} \mu_{\varepsilon}=0,
$$

and by definition of the weak two-scale convergence we have $\lim _{\varepsilon \rightarrow 0} \varepsilon \int_{\Omega} y_{\varepsilon}(x)\left(\nabla \varphi(x), b\left(\varepsilon^{-1} x\right)\right) \mathrm{d} \mu_{\varepsilon}=0$. Therefore, passing to the limit in (4.11), we conclude

$$
\lim _{\varepsilon \rightarrow 0} \int_{\Omega} a\left(\varepsilon^{-1} x\right) y_{\varepsilon}(x) \varphi \mathrm{d} \mu_{\varepsilon}=\int_{\Omega} \int_{\square} a(z) y(x, z) \mathrm{d} \mu(z) \varphi(x) \mathrm{d} x=0 .
$$

Due to the approximation Lemma [36] and the ergodicity property of the measure $\mu$ (see Thm. 4.3) the set of all functions $a \in \mathrm{L}^{2}(\square, \mathrm{d} \mu)$ satisfying condition (4.8), where $b$ are vectors from $\left(\mathrm{L}^{2}(\square, \mathrm{d} \mu)\right)^{2}$, is dense in the subspace of functions in $\mathrm{L}^{2}(\square, \mathrm{d} \mu)$ with mean value zero. Thus, from (4.12) we immediately conclude that $y(x, z)=y(x)$, i.e. the weak two-scale limit $y(x, z)$ in $(4.7)$ is independent of $z$.

Let us show now that condition (4.5) is satisfied. For this we consider the equality (4.9) with any vector $b \in V_{\text {sol }}$, i.e. $b \perp V_{\text {pot. }}$. This yields

$$
\int_{\Omega} \varphi(x)\left(\nabla y_{\varepsilon}(x), b\left(\varepsilon^{-1} x\right)\right) \mathrm{d} \mu_{\varepsilon}=-\int_{\Omega} y_{\varepsilon}(x)\left(\nabla \varphi, b\left(\varepsilon^{-1} x\right)\right) \mathrm{d} \mu_{\varepsilon} \quad \forall \varphi \in \mathrm{C}_{0}^{\infty}(\Omega) .
$$

Then passing in (4.13) to the limit (in the sense of two-scale convergence) and using (4.7), we obtain

$$
\int_{\Omega}\left[\int_{\square}(p(x, y), b(z)) \mathrm{d} \mu(z)\right] \varphi(x) \mathrm{d} x=-\int_{\Omega}\left(y(x) \int_{\square} b(z) \mathrm{d} \mu(z), \nabla \varphi(x)\right) \mathrm{d} x .
$$

Note that since $p \in \mathrm{L}^{2}(\Omega, \square)$, it follows that the function $v(x)=\int_{\square}(p(x, z), b(z)) \mathrm{d} \mu(x)$ belongs to $\mathrm{L}^{2}(\Omega)$. Let us set $\Theta(x)=y(x) \int_{\square} b(z) \mathrm{d} \mu(z)$. Then rewriting (4.14) in the form $\int_{\Omega} v \cdot \varphi \mathrm{d} x=-\int_{\Omega}(\Theta, \nabla \varphi) \mathrm{d} x$ and integrating by parts the expression on the right-hand side, we conclude that $v(x)=\left(\nabla y(x), \int_{\square} b(z) \mathrm{d} \mu(z)\right)$. Now we may use the non-degeneracy property of measure $\mu$ (Thm. 4.3). As a result, for $\eta=\int_{\square} b(z) \mathrm{d} \mu(z)$ can be 
fined a vector $q \in V_{\text {pot }}\left(V_{\text {pot }} \subset \mathrm{L}^{2}(\square)\right)$ such that $\eta=\int_{\square} q(z) \mathrm{d} \mu(z)$. Therefore $v(x)=\left(\nabla y(x), \int_{\square} q(z) \mathrm{d} \mu(z)\right)$ and we can give the following conclusion: since $\left(\nabla y, \int_{\square} q \mathrm{~d} \mu\right) \in \mathrm{L}^{2}(\Omega)$ in the sense of distributions and $q \in V_{\text {pot }}$, it follows that $\nabla y \in \mathrm{L}^{2}(\Omega)^{2}$ in the sense of distribution as well. Hence

$$
y \in \mathrm{L}^{2}(\Omega), \nabla y \in \mathrm{L}^{2}(\Omega)^{2} \Rightarrow y \in \mathrm{H}^{1}(\Omega) .
$$

In view of this, equation (4.14) can be rewritten in the form

$$
\int_{\Omega}\left(\int_{\square}(p(x, z), b(z)) \mathrm{d} \mu(z)\right) \varphi(x) \mathrm{d} x=\int_{\Omega}\left(\nabla y, \int_{\square} b(z) \mathrm{d} \mu(z)\right) \varphi(x) \mathrm{d} x,
$$

i.e.

$$
\int_{\Omega} \int_{\square}(p(x, z)-\nabla y(x), b(z)) \mathrm{d} \mu(z) \varphi(x) \mathrm{d} x=0 \quad \text { for every } \varphi \in \mathrm{C}_{0}^{\infty}(\Omega), b \in V_{\text {sol }} .
$$

Since the linear span of the vector-valued functions $\varphi(x) b(z)$ is dense in $\mathrm{L}^{2}\left(\Omega, V_{\text {sol }}\right)$ and the orthogonal decomposition (4.3) holds, it leads that to the inclusion $p(x, z)-\nabla y(x) \in \mathrm{L}^{2}\left(\Omega, V_{\mathrm{pot}}\right)$. This concludes the proof.

\section{VARIATIONAL CONVERGENCE OF CONSTRAined MiNimizATION PROBLEMS ON VARYING GRAPHS}

The main object in this section is the following sequence of constrained minimization problems

$$
\left\{\left\langle\inf _{(y, u, h) \in \Xi_{\varepsilon}} I_{\varepsilon}(y, u, h)\right\rangle, \quad \varepsilon \rightarrow 0\right\}, \text { where } I_{\varepsilon}: \Xi_{\varepsilon} \rightarrow \mathbb{R}, \Xi_{\varepsilon} \subset \mathbb{Z}_{\varepsilon}\left(\Omega, \Gamma_{1}\right) \quad \forall \varepsilon \in E .
$$

Definition 5.1. We say that the space $\mathrm{L}^{2}(\Omega)$ possesses the weak approximation property with respect to the family of Borel measures $\left\{\mu_{\varepsilon}\right\}_{\varepsilon \in E}$, if for every $\delta>0$ and every $u \in \mathrm{L}^{2}(\Omega)$ there exist an element $u^{*} \in \mathrm{L}^{2}(\Omega)$ and a sequence $\left\{u_{\varepsilon} \in \mathrm{L}^{2}\left(\Omega, \mathrm{d} \mu_{\varepsilon}\right)\right\}_{\varepsilon \in E}$ such that

$$
\left\|u-u^{*}\right\|_{\mathrm{L}^{2}(\Omega)} \leq \delta \quad \text { and } \quad u_{\varepsilon} \rightarrow u^{*} \quad \text { in } \mathrm{L}^{2}\left(\Omega, \mathrm{d} \mu_{\varepsilon}\right) .
$$

In this case the sequence $\left\{u_{\varepsilon} \in \mathrm{L}^{2}\left(\Omega, \mathrm{d} \mu_{\varepsilon}\right)\right\}_{\varepsilon \in E}$ is called $\delta$-realizing.

Obviously, we have the analogous notion of the weak approximation property for the space $\mathrm{L}^{2}\left(\Gamma_{1}\right)$ with respect to the family of Borel measures $\left\{\mu_{\varepsilon}^{S}\right\}$.

Lemma 5.2. The weak approximation property for the $\mathrm{L}^{2}(\Omega)$ with respect to the family of Borel measures $\left\{\mu_{\varepsilon}\right\}_{\varepsilon \in E}$ holds true.

Proof. Let $u$ be any element of $\mathrm{L}^{2}(\Omega)$. Since the inclusion $\mathrm{C}_{0}^{\infty}\left(\mathbb{R}^{2}\right) \subset \mathrm{L}^{2}(\Omega)$ is dense with respect to the strong topology for $\mathrm{L}^{2}(\Omega)$, it follows that for a given value $\delta>0$ there is an element $u^{*} \in \mathrm{C}_{0}^{\infty}\left(\mathbb{R}^{2}\right)$ such that $\left\|u-u^{*}\right\|_{L^{2}(\Omega)} \leq \delta$. Let us construct the $\delta$-realizing sequence as follows: $u_{\varepsilon}=u^{*}$ for every $\varepsilon>0$. Then due to the weak convergence of the measures $\mathrm{d} \mu_{\varepsilon} \rightarrow \mathrm{d} x$ we have

$$
\lim _{\varepsilon \rightarrow 0} \int_{\Omega} u_{\varepsilon} \varphi \psi \mathrm{d} \mu_{\varepsilon}=\int_{\Omega} u^{*} \varphi \psi \mathrm{d} x \forall \varphi \in \mathrm{C}_{0}^{0}(\Omega), \quad \lim _{\varepsilon \rightarrow 0} \int_{\Omega}\left(u_{\varepsilon}\right)^{2} \mathrm{~d} \mu_{\varepsilon}=\int_{\Omega}\left(u^{*}\right)^{2} \mathrm{~d} x .
$$


Hence, by the criterium of strong convergence in $\mathrm{L}^{2}\left(\Omega, \mathrm{d} \mu_{\varepsilon}\right)$, we obtain $u_{\varepsilon} \rightarrow u^{*}$ in $\mathrm{L}^{2}\left(\Gamma_{1}, \mathrm{~d} \mu_{\varepsilon}^{S}\right)$.

An analogous result holds for the space $\mathrm{L}^{2}\left(\Gamma_{1}\right)$.

Definition 5.3. We say that a set $\Xi_{0} \subset \mathbb{Y}_{0}=H^{1}(\Omega) \times \mathrm{L}^{2}(\Omega) \times \mathrm{L}^{2}\left(\Gamma_{1}\right)$ is the sequential two-scale limit, or K-limit, of the sequence

$$
\left\{\Xi_{\varepsilon} \subset \mathbb{Z}_{\varepsilon}\left(\Omega, \Gamma_{1}\right) \equiv V\left(\Omega, \Gamma_{2}, \mathrm{~d} \mu_{\varepsilon}\right) \times \mathrm{L}^{2}\left(\Omega, \mathrm{d} \mu_{\varepsilon}\right) \times \mathrm{L}^{2}\left(\Gamma_{1}, \mathrm{~d} \mu_{\varepsilon}^{S}\right)\right\}_{\varepsilon \in E}
$$

if the following conditions are satisfied:

(i) for every triplet $(y, u, h) \in \Xi_{0}$ and any value $\delta>0$ there exist a constant $\varepsilon^{0} \in E$ and a $\delta$-realizing sequence $\left\{\left(y_{\varepsilon}, u_{\varepsilon}, h_{\varepsilon}\right)\right\}_{\varepsilon \in E}$ such that

$$
\left(y_{\varepsilon}, u_{\varepsilon}, h_{\varepsilon}\right) \in \Xi_{\varepsilon} \forall \varepsilon \leq \varepsilon^{0}, \quad\left(y_{\varepsilon}, u_{\varepsilon}, h_{\varepsilon}\right) \stackrel{w}{\longrightarrow}(\widehat{y}, \widehat{u}, \widehat{h}), \quad\|(y, u, h)-(\widehat{y}, \widehat{u}, \widehat{h})\|_{\mathbb{Y}_{0}} \leq \delta
$$

(ii) if $\left\{\Xi_{\varepsilon_{k}}\right\}$ is a subsequence of $\left\{\Xi_{\varepsilon}\right\}_{\varepsilon \in E}$ and $\left\{\left(y_{k}, u_{k}, h_{k}\right)\right\}$ is a sequence $w$-converging to $(y, u, h)$ such that $\left(y_{k}, u_{k}, h_{k}\right) \in \Xi_{\varepsilon_{k}}$ for every $k \in \mathbb{N}$, then $(y, u, h) \in \Xi_{0}$.

Remark 5.4. Note also that if $\mathrm{d} \mu_{\varepsilon}=\mathrm{d} x, \delta=0$, and $\mathrm{d} \mu_{\varepsilon}^{S}=\mathrm{d} l$ in (5.4) then the notion of the $K$-limit set coincides with the well-known notion of the sequential topological limit in the sense of Kuratowski with respect to the product of weak topologies in $\mathrm{H}^{1}(\Omega), \mathrm{L}^{2}(\Omega)$, and $\mathrm{L}^{2}\left(\Gamma_{1}\right)$, respectively (see [12]).

Definition 5.5. We say that a minimization problem

$$
\left\langle\inf _{(y, u, h) \in \Xi_{0}} I_{0}(y, u, h)\right\rangle
$$

is the variational limit of the sequence (5.1) with respect to the $w$-convergence if the following conditions are satisfied:

(i) $\Xi_{0} \subset \mathbb{Y}_{0}$ is a non-empty $K$-limit of the sets $\left\{\Xi_{\varepsilon}\right\}_{\varepsilon \in E}$;

(ii) for every triplet $(y, u, h) \in \Xi_{0}$ and for every sequence $\left\{\left(y_{k}, u_{k}, h_{k}\right)\right\}_{k \in \mathbb{N}} w$-converging to $(y, u, h)$ such that $\left(y_{k}, u_{k}, h_{k}\right) \in \Xi_{\varepsilon_{k}}$, where $\varepsilon_{k} \rightarrow 0$ as $k \rightarrow \infty$, it is

$$
I_{0}(y, u, h) \leq \liminf _{k \rightarrow \infty} I_{\varepsilon_{k}}\left(y_{k}, u_{k}, h_{k}\right)
$$

(iii) for every triplet $(y, u, h) \in \Xi_{0}$ and any $\delta>0$ there are a constant $\varepsilon^{0} \in E$ and a $\delta$-realizing sequence $\left\{\left(y_{\varepsilon}, u_{\varepsilon}, h_{\varepsilon}\right)\right\}_{\varepsilon \in E}$ such that

$$
\begin{gathered}
\left(y_{\varepsilon}, u_{\varepsilon}, h_{\varepsilon}\right) \in \Xi_{\varepsilon} \forall \varepsilon \leq \varepsilon^{0}, \quad\left(y_{\varepsilon}, u_{\varepsilon}, h_{\varepsilon}\right) \stackrel{w}{\longrightarrow}(\widehat{y}, \widehat{u}, \widehat{h}), \quad\|(y, u, h)-(\widehat{y}, \widehat{u}, \widehat{h})\|_{\mathbb{Y}_{0}} \leq \delta \\
I_{0}(y, u, h) \geq \limsup _{\varepsilon \rightarrow 0} I_{\varepsilon}\left(y_{\varepsilon}, u_{\varepsilon}, h_{\varepsilon}\right)-\widehat{C} \delta
\end{gathered}
$$

with some constant $\widehat{C}>0$ independent of $\delta$.

Remark 5.6. Note that this definition can be interpreted as the natural extension of the well-known notion of sequential $\Gamma$-convergence. We do not want to dwell on details of $\Gamma$-convergence theory, but we do want to emphasize that the weak variational limit in the sense of Definition 5.5 possesses the fine variational properties that are similar to that of $\Gamma$-limit.

Theorem 5.7. Assume that the constrained minimization problem (5.5) is the variational limit of the sequence (5.1) in the sense of Definition 5.3 and this problem has a unique solution $\left(y^{0}, u^{0}, h^{0}\right)$. For every 
$\varepsilon \in E$, let $\left(y_{\varepsilon}^{0}, u_{\varepsilon}^{0}, h_{\varepsilon}^{0}\right) \in \Xi_{\varepsilon}$ be an optimal solution of the corresponding problem (3.3)-(3.6). If the sequence $\left\{\left(y_{\varepsilon}^{0}, u_{\varepsilon}^{0}, h_{\varepsilon}^{0}\right)\right\}_{\varepsilon \in E}$ is relatively $w$-compact then

$$
\begin{gathered}
\left(y_{\varepsilon}^{0}, u_{\varepsilon}^{0}, h_{\varepsilon}^{0}\right) \stackrel{w}{\longrightarrow}\left(y^{0}, u^{0}, h^{0}\right), \\
\inf _{(y, u, h) \in \Xi_{0}} I_{0}(y, u, h)=I_{0}\left(y^{0}, u^{0}, h^{0}\right)=\lim _{\varepsilon \rightarrow 0} I_{\varepsilon}\left(y_{\varepsilon}^{0}, u_{\varepsilon}^{0}, h_{\varepsilon}^{0}\right)=\lim _{\varepsilon \rightarrow 0} \inf _{\left(y_{\varepsilon}, u_{\varepsilon}, h_{\varepsilon}\right) \in \Xi_{\varepsilon}} I_{\varepsilon}\left(y_{\varepsilon}, u_{\varepsilon}, h_{\varepsilon}\right) .
\end{gathered}
$$

Proof. Let $\left\{\left(y_{\varepsilon_{k}}^{0}, u_{\varepsilon_{k}}^{0}, h_{\varepsilon_{k}}^{0}\right)\right\}_{k \in \mathbb{N}}$ be a subsequence of the sequence of minimizers $w$-converging to some triplet $\left(y^{*}, u^{*}, h^{*}\right)$. Then by Definition 5.3, we have $\left(y^{*}, u^{*}, h^{*}\right) \in \Xi_{0}$. Moreover, due to the properties (ii) of Definition 5.5 , one gets

$$
\liminf _{k \rightarrow \infty} \min _{(y, u, h) \in \Xi_{\varepsilon_{k}}} I_{\varepsilon_{k}}(y, u, h)=\liminf _{k \rightarrow \infty} I_{\varepsilon_{k}}\left(y_{\varepsilon_{k}}^{0}, u_{\varepsilon_{k}}^{0}, h_{\varepsilon_{k}}^{0}\right) \geq I_{0}\left(y^{*}, u^{*}, h^{*}\right) \geq \min _{(y, u, h) \in \Xi_{0}} I_{0}(y, u, h) .
$$

Let $\left(y^{0}, u^{0}, h^{0}\right) \in \Xi_{0}$ be an optimal solution of the limit problem (5.5). Let us fix a value $\delta>0$. Then, by property (ii) of Definition 5.5 there exist a $\delta$-realizing sequence $\left\{\left(\widehat{y}_{\varepsilon}, \widehat{u}_{\varepsilon}, \widehat{h}_{\varepsilon}\right) \in \Xi_{\varepsilon}\right\}_{\varepsilon \rightarrow 0}$ such that

$$
\left(\widehat{y}_{\varepsilon}, \widehat{u}_{\varepsilon}, \widehat{h}_{\varepsilon}\right) \stackrel{w}{\rightarrow}(\widehat{y}, \widehat{u}, \widehat{h}), \quad\left\|\left(y^{0}, u^{0}, h^{0}\right)-(\widehat{y}, \widehat{u}, \widehat{h})\right\|_{\mathbb{Y}_{0}} \leq \delta, \quad \text { and } \quad I_{0}\left(y^{0}, u^{0}, h^{0}\right) \geq \limsup _{\varepsilon \rightarrow 0} I_{\varepsilon}\left(\widehat{y}_{\varepsilon}, \widehat{u}_{\varepsilon}, \widehat{h}_{\varepsilon}\right)-\widehat{C} \delta .
$$

Using this fact we have

$$
\begin{aligned}
\min _{(y, u, h) \in \Xi_{0}} I_{0}(y, u, h)+\widehat{C} \delta=I_{0}\left(y^{0}, u^{0}, h^{0}\right) & +\widehat{C} \delta \geq \limsup _{\varepsilon \rightarrow 0} I_{\varepsilon}\left(\widehat{y}_{\varepsilon}, \widehat{u}_{\varepsilon}, \widehat{h}_{\varepsilon}\right) \geq \limsup _{\varepsilon \rightarrow 0} \min _{(y, u, h) \in \Xi_{\varepsilon}} I_{\varepsilon}(y, u, h) \\
& \geq \limsup _{k \rightarrow \infty} \min _{(y, u, h) \in \Xi_{\varepsilon_{k}}} I_{\varepsilon_{k}}(y, u, h)=\limsup _{k \rightarrow \infty} I_{\varepsilon_{k}}\left(y_{\varepsilon_{k}}^{0}, u_{\varepsilon_{k}}^{0}, h_{\varepsilon_{k}}^{0}\right) .
\end{aligned}
$$

From this and (5.10) we deduce that

$$
\liminf _{k \rightarrow \infty} I_{\varepsilon_{k}}\left(y_{\varepsilon_{k}}^{0}, u_{\varepsilon_{k}}^{0}, h_{\varepsilon_{k}}^{0}\right) \geq \limsup _{k \rightarrow \infty} I_{\varepsilon_{k}}\left(y_{\varepsilon_{k}}^{0}, u_{\varepsilon_{k}}^{0}, h_{\varepsilon_{k}}^{0}\right)-\widehat{C} \delta .
$$

Since this inequality holds true for any sufficiently small $\delta>0$ it follows that combining the above obtained relations (5.10) and (5.11) we get

$$
I_{0}\left(y^{*}, u^{*}, h^{*}\right)=I_{0}\left(y^{0}, u^{0}, h^{0}\right)=\min _{(y, u, h) \in \Xi_{0}} I_{0}(y, u, h), \quad I_{0}\left(y^{0}, u^{0}, h^{0}\right)=\lim _{k \rightarrow \infty} \min _{(y, u, h) \in \Xi_{\varepsilon_{k}}} I_{\varepsilon_{k}}(y, u, h) .
$$

Using these relations and the fact that an optimal triplet for the problem (5.5) is unique, we obtain

$$
\left(y^{*}, u^{*}, h^{*}\right)=\left(y^{0}, u^{0}, h^{0}\right) .
$$

Since this equality holds for the $w$-limits of all subsequences of $\left\{\left(y_{\varepsilon}^{0}, u_{\varepsilon}^{0}, h_{\varepsilon}^{0}\right)\right\}_{\varepsilon \in E}$, it follows that these limits are coincident and therefore $\left(y^{0}, u^{0}, h^{0}\right)$ is the $w$-limit of the whole sequence $\left\{\left(y_{\varepsilon}^{0}, u_{\varepsilon}^{0}, h_{\varepsilon}^{0}\right)\right\}_{\varepsilon \in E}$. Hence, making for the sequence of minimizers what we did before with a subsequence $\left\{\left(y_{\varepsilon_{k}}^{0}, u_{\varepsilon_{k}}^{0}, h_{\varepsilon_{k}}^{0}\right)\right\}_{k \in \mathbb{N}}$, we have

$$
\begin{aligned}
& \liminf _{\varepsilon \rightarrow 0} \min _{(y, u, h) \in \Xi_{\varepsilon}} I_{\varepsilon}(y, u, h)=\liminf _{\varepsilon \rightarrow 0} I_{\varepsilon}\left(y_{\varepsilon}^{0}, u_{\varepsilon}^{0}, h_{\varepsilon}^{0}\right) \geq I_{0}\left(y^{0}, u^{0}, h^{0}\right)=\min _{(y, u, h) \in \Xi_{0}} I_{0}(y, u, h) \\
& \quad \geq \limsup _{\varepsilon \rightarrow 0} I_{\varepsilon}\left(\widehat{y}_{\varepsilon}, \widehat{u}_{\varepsilon}, \widehat{h}_{\varepsilon}\right)-\delta \geq \limsup _{\varepsilon \rightarrow 0} \min _{(y, u, h) \in \Xi_{\varepsilon}} I_{\varepsilon}(y, u, h)-\widehat{C} \delta=\limsup _{\varepsilon \rightarrow 0} I_{\varepsilon}\left(y_{\varepsilon}^{0}, u_{\varepsilon}^{0}, h_{\varepsilon}^{0}\right)-\widehat{C} \delta \quad \forall \delta>0 .
\end{aligned}
$$

Thus we have obtained the required conclusion. This proof is complete. 


\section{Homogenization of the optimal CONTROL PROBlems ON $\varepsilon$ PERIODIC GRAPHS}

We begin with the so-called "convergence property of gradients of arbitrary solutions". This property can be viewed as a natural requirement on the homogenized matrix to the family of matrix $A_{\varepsilon}$ in $(3.5)$. Let $\left\{A_{\varepsilon}(x) \in\right.$ $\left.\mathcal{L}\left(\mathbb{R}^{2}, \mathbb{R}^{2}\right)\right\}_{\varepsilon \in \mathbb{N}}$ be a family of square $\mu_{\varepsilon}$-measurable matrices satisfying the inequality (3.2) for every $\varepsilon \in E$.

Definition 6.1. (convergence of gradients of arbitrary solutions). We say that a matrix $A^{\text {hom }}(x) \in \mathcal{L}\left(\mathbb{R}^{2}, \mathbb{R}^{2}\right)$ is the homogenized matrix with respect to the family $\left\{A_{\varepsilon}(x)\right\}$ as $\varepsilon$ tends to zero, if:

(a) $A^{\text {hom }} \in\left[\mathrm{L}^{\infty}(\Omega)\right]^{2 \times 2}, A^{\text {hom }}(x)$ is coercive;

(b) $A_{\varepsilon} \nabla y_{\varepsilon} \rightarrow A^{\text {hom }} \nabla y_{0}$ in $\mathrm{L}^{2}\left(\Omega, \mathrm{d} \mu_{\varepsilon}\right)^{2}$ for every sequence $\left\{\left(y_{\varepsilon}, u_{\varepsilon}, h_{\varepsilon}\right) \in \mathbb{Z}_{\varepsilon}\left(\Omega, \Gamma_{1}\right)\right\}_{\varepsilon \in E}$ such that $\left(y_{\varepsilon}, \nabla y_{\varepsilon}\right)$ satisfy the relation $(3.5)$, and $\left(y_{\varepsilon}, u_{\varepsilon}, h_{\varepsilon}\right) \stackrel{w}{\rightarrow}\left(y_{0}, u_{0}, h_{0}\right)$.

In order to find a homogenized problem to the family (3.3)-(3.6) we introduce two auxiliary sets

$$
\begin{aligned}
& \operatorname{Graph}\left(\mathbb{P}_{\varepsilon}\right)=\left\{(y, u, h) \in \mathbb{Z}_{\varepsilon}\left(\Omega, \Gamma_{1}\right):\right. \\
& \left.\qquad \int_{\Omega}\left[\left(A_{\varepsilon}(x) \nabla y, \nabla \varphi\right)+\alpha y \varphi\right] \mathrm{d} \mu_{\varepsilon}=\int_{\Omega} u \varphi \mathrm{d} \mu_{\varepsilon}+\int_{\Gamma_{1}} h \varphi \mathrm{d} \mu_{\varepsilon}^{S}, \forall \varphi \in \mathrm{C}^{\infty}\left(\Omega, \Gamma_{2}\right)\right\}, \quad(6.1) \\
& \operatorname{Graph}\left(\mathbb{P}^{\text {hom }}\right)=\left\{(y, u, h) \in \mathbb{Z}_{0}\left(\Omega, \Gamma_{1}\right) \equiv \mathrm{H}^{1}\left(\Omega, \Gamma_{2}\right) \times \mathrm{L}^{2}(\Omega) \times \mathrm{L}^{2}\left(\Gamma_{1}\right):\right. \\
& \left.\int_{\Omega}\left[\left(A^{\text {hom }} \nabla y, \nabla \varphi\right)+\alpha y \varphi\right] \mathrm{d} x=\int_{\Omega} u \varphi \mathrm{d} x+\int_{\Gamma_{1}} h \varphi \mathrm{d} l, \forall \mathrm{C}^{\infty}\left(\Omega, \Gamma_{2}\right)\right\}
\end{aligned}
$$

and make use the following lemmas.

Lemma 6.2. Assume that for the family $\left\{A_{\varepsilon} \in \mathcal{L}\left(\mathbb{R}^{2}, \mathbb{R}^{2}\right)\right\}$ there exists a homogenized matrix in the sense of Definition 6.1. Then the sequence of sets $\left\{\Xi_{\varepsilon}\right\}_{\varepsilon \in E}$ is $K$-convergent to the set $\Xi_{0}$, where

$$
\begin{aligned}
\Xi_{0}=\left\{(y, u, h) \in \mathbb{Z}_{0}\left(\Omega, \Gamma_{1}\right):\right. & |u| \leq c_{u} \text { a.e. in } \Omega, \quad|h| \leq c_{h} \text { a.e. on } \Gamma_{1}, \\
& \left.\int_{\Omega}\left[\left(A^{\mathrm{hom}} \nabla y, \nabla \varphi\right)+\alpha y \varphi\right] \mathrm{d} x=\int_{\Omega} u \varphi \mathrm{d} x+\int_{\Gamma_{1}} h \varphi \mathrm{d} l, \forall \mathrm{C}^{\infty}\left(\Omega, \Gamma_{2}\right)\right\} .
\end{aligned}
$$

Proof. Let $\left(y^{0}, u^{0}, h^{0}\right)$ be any triplet of $\Xi_{0}$, and let us fix a value $\delta>0$. Since the space of smooth functions $\mathrm{C}^{\infty}(\Omega)$ is dense in $\mathrm{L}^{2}(\Omega)$, it follows that for a given value $\delta>0$ there is an element $u^{*} \in \mathrm{C}_{0}^{\infty}\left(\mathbb{R}^{2}\right)$ such that: $\left\|u^{0}-u^{*}\right\|_{L^{2}(\Omega)} \leq \delta$ and $\left|u^{*}\right| \leq c_{u}$ in $\Omega$. Let us construct a $\delta$-realizing sequence as follows: $u_{\varepsilon}=u^{*}$ for every $\varepsilon>0$. Then due to the weak convergence of the measures $\mathrm{d} \mu_{\varepsilon} \rightarrow \mathrm{d} x$, we have

$$
\int_{\Omega} \varphi u_{\varepsilon} \mathrm{d} \mu_{\varepsilon} \rightarrow \int_{\Omega} \varphi u^{*} \mathrm{~d} x \quad \text { for every } \varphi \in \mathrm{C}_{0}^{\infty}\left(\mathbb{R}^{2}\right)
$$

i.e. $\left\{u_{\varepsilon}\right\}$ is a $\delta$-realizing sequence of admissible controls for $u^{0}$. By analogy, we may choose the sequence $\left\{h_{\varepsilon} \in \mathrm{C}^{\infty}\left(\Gamma_{1}\right)\right\}$ such that

$$
h_{\varepsilon}=h^{*} \forall \varepsilon, \quad\left|h_{\varepsilon}\right| \leq c_{h} \mu^{S} \text {-a.e. on } \Gamma_{1}, \quad h_{\varepsilon} \in \mathrm{L}^{2}\left(\Gamma_{1}, \mathrm{~d} \mu_{\varepsilon}\right), \quad h_{\varepsilon} \rightarrow h^{*} \text { in } \mathrm{L}^{2}\left(\Gamma_{1}, \mathrm{~d} \mu_{\varepsilon}^{S}\right),
$$


where $\left\|h^{0}-h^{*}\right\|_{L^{2}\left(\Gamma_{1}\right)} \leq \delta$ and $\left|h^{*}\right| \leq c_{h}$ on $\Gamma_{1}$.

Let $\left\{y_{\varepsilon} \in V\left(\Omega, \Gamma_{2}, \mathrm{~d} \mu_{\varepsilon}\right)\right\}$ be the sequence of solution of the boundary value problem (3.4)-(3.5) under the corresponding control functions $u=u_{\varepsilon}$ and $h=h_{\varepsilon}$. Using the estimate (3.7) we find that

$$
\left\|y_{\varepsilon}\right\|_{\mathrm{L}^{2}\left(\Omega, \mathrm{d} \mu_{\varepsilon}\right)} \leq 2 \alpha^{-1} C,\left\|\nabla y_{\varepsilon}\right\|_{\left(\mathrm{L}^{2}\left(\Omega, \mathrm{d} \mu_{\varepsilon}\right)\right)^{2}} \leq 2 \alpha^{-1} C .
$$

Then, thanks to Remark 3.4 and Theorem 4.7 we may always suppose that the sequence of the triplets $\left\{\left(y_{\varepsilon}, u_{\varepsilon}, h_{\varepsilon}\right) \in \Xi_{\varepsilon}\right\}_{\varepsilon \in E}$ is $w$-convergent. Let $\left(y^{*}, u^{*}, h^{*}\right)$ be its $w$-limit. Our aim is to prove that $\left(y^{*}, u^{*}, h^{*}\right) \in$ $\operatorname{Graph}\left(\mathbb{P}^{\text {hom }}\right)$. In view of our assumptions and Definition 6.1 , we have

$$
\begin{array}{cl}
u_{\varepsilon} \rightarrow u^{*} \text { in } \mathrm{L}^{2}\left(\Omega, \mathrm{d} \mu_{\varepsilon}\right), & h_{\varepsilon} \rightarrow h^{*} \text { in } \mathrm{L}^{2}\left(\Gamma_{1}, \mathrm{~d} \mu_{\varepsilon}^{S}\right), \\
y_{\varepsilon} \stackrel{2}{\rightarrow} y^{*}(x), \quad y_{\varepsilon} \rightarrow y^{*} \text { in } \mathrm{L}^{2}\left(\Omega, \mathrm{d} \mu_{\varepsilon}\right), & A_{\varepsilon}(\cdot) \nabla y_{\varepsilon} \rightarrow A^{\mathrm{hom}} \nabla y^{*} \text { in } \mathrm{L}^{2}\left(\Omega, \mathrm{d} \mu_{\varepsilon}\right)^{2} .
\end{array}
$$

Therefore, passing to the limit as $\varepsilon \rightarrow 0$ in the integral identity

$$
\int_{\Omega}\left[\left(A_{\varepsilon}(x) \nabla y_{\varepsilon}, \nabla \varphi\right)+\alpha y_{\varepsilon} \varphi\right] \mathrm{d} \mu_{\varepsilon}=\int_{\Omega} u_{\varepsilon} \varphi \mathrm{d} \mu_{\varepsilon}+\int_{\Gamma_{1}} h_{\varepsilon} \varphi \mathrm{d} \mu_{\varepsilon}^{S}, \quad \forall \varphi \in \mathrm{C}^{\infty}\left(\Omega, \Gamma_{2}\right)
$$

we obtain

$$
\int_{\Omega}\left[\left(A^{\mathrm{hom}} \nabla y^{*}, \nabla \varphi\right)+\alpha y^{*} \varphi\right] \mathrm{d} x=\int_{\Omega} u^{*} \varphi \mathrm{d} x+\int_{\Gamma_{1}} h^{*} \varphi \mathrm{d} l, \quad \forall \varphi \in \mathrm{C}^{\infty}\left(\Omega, \Gamma_{2}\right) .
$$

Hence, we have $\left(y^{*}, u^{*}, h^{*}\right) \in \operatorname{Graph}\left(\mathbb{P}^{\text {hom }}\right)$. In view of the coerciveness property of the homogenized ma$\operatorname{trix} A^{\text {hom }}$ we have the standard a priori estimate

$$
\left\|y^{0}-y^{*}\right\|_{\mathrm{H}^{1}(\Omega)} \leq C\left[\left\|u^{0}-u^{*}\right\|_{\mathrm{L}^{2}(\Omega)}+\left\|h^{0}-h^{*}\right\|_{\mathrm{L}^{2}\left(\Gamma_{1}\right)}\right] \leq 2 \delta C .
$$

Thus, $\left\|\left(y^{0}, u^{0}, h^{0}\right)-\left(y^{*}, u^{*}, h^{*}\right)\right\|_{\mathbb{Y}_{0}} \leq 2 \max \{C, 1\} \delta$, that is, $\left\{\left(y_{\varepsilon}, u_{\varepsilon}, h_{\varepsilon}\right) \in \Xi_{\varepsilon}\right\}_{\varepsilon \in E}$ is a $\delta$-realizing sequence with the required properties. Thereby the following inclusion $K-\lim \left(\Xi_{\varepsilon}\right) \supseteq \Xi_{0}$ is established.

In order to obtain the inverse inclusion we consider a $w$-convergent sequence $\left\{\left(y_{k}, u_{k}, h_{k}\right)\right\}_{k \in \mathbb{N}}$ with the following properties:

(a) $\left(y_{k}, u_{k}, h_{k}\right) \in \Xi_{\varepsilon_{k}}$ for every $k \in \mathbb{N}$, where $\left\{\varepsilon_{k}\right\}$ is some subsequence of indices converging to zero as $k$ tends to $\infty$;

(b) $\left(y_{k}, u_{k}, h_{k}\right) \rightarrow\left(y^{*}, u^{*}, h^{*}\right)$ in the $w$-sense.

Then proceeding as in the previous part of this lemma we can show that

$$
\nabla y_{k} \stackrel{2}{\rightarrow} \nabla y^{*}+\mathbf{v}(x, z), \quad \mathbf{v} \in \mathrm{L}^{2}\left(\Omega, V_{\mathrm{pot}}\right), \quad A_{\varepsilon_{k}}(\cdot) \nabla y_{k} \rightarrow A^{\mathrm{hom}} \nabla y^{*} \text { in } \mathrm{L}^{2}\left(\Omega, \mathrm{d} \mu_{\varepsilon}\right)^{2} .
$$

Therefore, passing to the limit in the integral identity

$$
\int_{\Omega}\left[\left(A_{\varepsilon_{k}}(x) \nabla y_{k}, \nabla \varphi\right)+\alpha y_{k} \varphi\right] \mathrm{d} \mu_{\varepsilon_{k}}=\int_{\Omega} u_{k} \varphi \mathrm{d} \mu_{\varepsilon_{k}}+\int_{\Gamma_{1}} h_{k} \varphi \mathrm{d} \mu_{\varepsilon_{k}}^{S} \quad \forall \varphi \in \mathrm{C}^{\infty}\left(\Omega, \Gamma_{2}\right),
$$

it leads us to the relation (6.3). Hence

$$
\left(y^{*}, u^{*}, h^{*}\right) \in \operatorname{Graph}\left(\mathbb{P}^{\text {hom }}\right) .
$$

Hence it remains to show that the limit control functions $u^{*} \in \mathrm{L}^{2}(\Omega)$ and $h^{*} \in \mathrm{L}^{2}\left(\Gamma_{1}\right)$ satisfy the corresponding constraints

$$
\left|u^{*}\right| \leq c_{u} \text { a.e. in } \Omega, \quad\left|h^{*}\right| \leq c_{h} \text { a.e. on } \Gamma_{1} .
$$


Indeed, for every $k \in \mathbb{N}$ and every positive function $\varphi \in \mathrm{C}^{\infty}\left(\Omega, \Gamma_{2}\right)$ we have

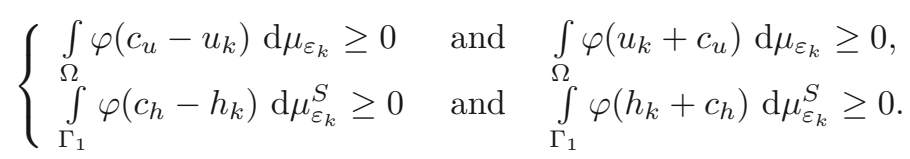

Using the facts that $\mathrm{d} \mu_{\varepsilon} \rightarrow \mathrm{d} x$ and $\mathrm{d} \mu_{\varepsilon}^{S} \rightarrow \mathrm{d} l$, and passing the limit in (6.6) as $k \rightarrow \infty$ one gets

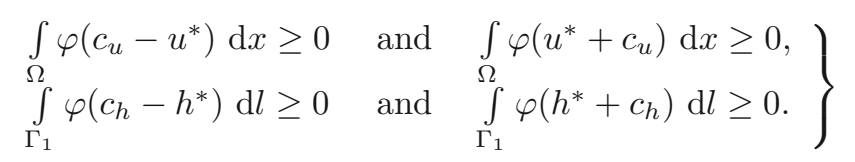

Since $\varphi$ in (6.7) is an arbitrary positive function if follows that the inequalities (6.5) hold true. In a result, combining (6.4) and (6.5) we deduce: $\left(y^{*}, u^{*}, h^{*}\right) \in \Xi_{0}$. Thus we have obtained the required inclusion $K-$ $\lim \Xi_{\varepsilon} \subseteq \Xi_{0}$, that concludes the proof.

Corollary 6.3. Suppose that the matrix $A_{\varepsilon}(x)$ is defined as $A_{\varepsilon}(x)=A\left(\varepsilon^{-1} x\right)$, where $A(z)$ is a $\square$-periodic $\mu$-measurable matrix satisfying condition (3.2). Then the limiting matrix $A^{\text {hom }}$ can be defined as

$$
A^{\mathrm{hom}} \xi=\int_{\square} A(z)\left(\xi+v^{0}\right) \mathrm{d} \mu(z),
$$

where $v^{0} \in V_{\mathrm{pot}}$ is the solution of the following problem

$$
\min _{v \in V_{\mathrm{pot}}} \int_{\square}(\xi+v, A(\xi+v)) \mathrm{d} \mu=\int_{\square}\left(\xi+v^{0}, A\left(\xi+v^{0}\right)\right) \mathrm{d} \mu .
$$

Proof. Let $\left\{\left(y_{\varepsilon}, u_{\varepsilon}, h_{\varepsilon}\right) \in \Xi_{\varepsilon}\right\}_{\varepsilon \in E}$ be a $w$-convergent sequence, and let $\left(y^{*}, u^{*}, h^{*}\right)$ be its $w$-limit. Then

$$
\nabla y_{\varepsilon} \stackrel{2}{\rightarrow} \nabla y^{*}+\mathbf{v}(x, z),
$$

where $\mathbf{v} \in \mathrm{L}^{2}\left(\Omega, V_{\text {pot }}\right)$. Emphasize that the gradients in (6.10) are uniquely defined (Lem. 3.3). For this we consider the integral identity (3.5) with the test function $\varphi(x)=\varepsilon \Psi(x) \omega\left(\varepsilon^{-1} x\right)$, where $\Psi \in \mathrm{C}^{\infty}\left(\Omega, \Gamma_{2}\right)$, and $\omega \in \mathrm{C}_{\mathrm{per}}^{\infty}(\square)$. This yields

$$
\begin{aligned}
\int_{\Omega}\left(A\left(\varepsilon^{-1} x\right) \nabla y_{\varepsilon}, \nabla_{z} \omega\right) \Psi \mathrm{d} \mu_{\varepsilon}+\varepsilon \int_{\Omega}\left(A\left(\varepsilon^{-1} x\right) \nabla y_{\varepsilon}, \nabla \Psi\right) \omega \mathrm{d} \mu_{\varepsilon}+\varepsilon \int_{\Omega} \alpha y_{\varepsilon} \Psi \omega \mathrm{d} \mu_{\varepsilon}= \\
\varepsilon \int_{\Omega} u_{\varepsilon} \Psi \omega \mathrm{d} \mu_{\varepsilon}+\varepsilon \int_{\Gamma_{1}} h_{\varepsilon} \Psi \omega \mathrm{d} \mu_{\varepsilon}^{S} .
\end{aligned}
$$

It is easy to see that after passing to the limit as $\varepsilon \rightarrow 0$ we obtain

$$
\lim _{\varepsilon \rightarrow 0} \int_{\Omega}\left(A\left(\varepsilon^{-1} x\right) \nabla y_{\varepsilon}, \nabla_{z} \omega\left(\varepsilon^{-1}\right)\right) \Psi(x) \mathrm{d} \mu_{\varepsilon}=0 .
$$

In view of (6.10) and the definition of the weak two-scale limit we have

$$
\lim _{\varepsilon \rightarrow 0} \int_{\Omega} \Psi(x)\left[\omega\left(\varepsilon^{-1} x\right) A\left(\varepsilon^{-1} x\right)\right] \nabla y_{\varepsilon}(x) \mathrm{d} \mu_{\varepsilon}=\int_{\Omega} \int_{\square} \Psi(x) \omega(z) A(z)\left[\nabla y^{*}(x)+\mathbf{v}(x, z)\right] \mathrm{d} \mu(z) \mathrm{d} x .
$$


Therefore

$$
\begin{aligned}
& A\left(\varepsilon^{-1} x\right) \nabla y_{\varepsilon}(x) \stackrel{2}{\rightarrow} \quad A(z)\left[\nabla y^{*}(x)+\mathbf{v}(x, z)\right] \\
& A\left(\varepsilon^{-1} x\right) \nabla y_{\varepsilon}(x) \rightarrow \int_{\square} A(z)\left[\nabla y^{*}(x)+\mathbf{v}(x, z)\right] \mathrm{d} \mu(z) \quad \text { in } \mathrm{L}^{2}\left(\Omega, \mathrm{d} \mu_{\varepsilon}\right)^{2} .
\end{aligned}
$$

As a result, we can rewrite (6.11) in the explicit form

$$
\lim _{\varepsilon \rightarrow 0} \int_{\Omega}\left(A\left(\varepsilon^{-1} x\right) \nabla y_{\varepsilon}, \nabla_{z} \omega\right) \Psi \mathrm{d} \mu_{\varepsilon}=\int_{\Omega} \int_{\square}\left(A(z)\left[\nabla y^{*}+\mathbf{v}(x, z)\right], \nabla_{z} \omega\right) \mathrm{d} \mu(z) \Psi(x) \mathrm{d} x=0 .
$$

Since this equality holds true for every $\Psi \in \mathrm{C}^{\infty}\left(\Omega, \Gamma_{2}\right)$ this means that

$$
\int_{\square}\left(A\left(\nabla y^{*}+\mathbf{v}\right), p\right) \mathrm{d} \mu=0 \quad \forall p \in \mathrm{C}_{\mathrm{per}}^{\infty}(\square)^{2} .
$$

However, as follows from (6.9), the equality (6.13) can be viewed as Euler's equation for the minimum problem (6.9). Since this problem has a unique solution $\mathbf{v}^{0}$ it follows that $\mathbf{v}^{0}$ is the unique solution of (6.13) as well. Thus, putting $\mathbf{v}=\mathbf{v}^{0}$ in (6.12) and using formula (6.8) we immediately deduce that

$$
A\left(\varepsilon^{-1} x\right) \nabla y_{\varepsilon} \rightarrow A^{\text {hom }} \nabla y^{*} \quad \text { in } L^{2}\left(\Omega, \mathrm{d} \mu_{\varepsilon}\right)^{2} .
$$

Lemma 6.4. Under the assumptions of Lemma 6.2 the limit functional $I_{0}: \Xi_{0} \rightarrow \mathbb{R}$ in (3.12) has the following representation

$$
I_{0}(y, u, h)=k_{1} \int_{\Omega}\left(y-z_{d}\right)^{2} \mathrm{~d} x+k_{2} \int_{\Omega} u^{2} \mathrm{~d} x+k_{3} \int_{\Gamma_{1}} h^{2} \mathrm{~d} l .
$$

Proof. To prove the representation (6.14) we have to verify the conditions (ii)-(iii) of Definition 5.3. Let ( $y, u, h$ ) be any triplet of $\Xi_{0}$, and let $\left\{\left(y_{k}, u_{k}, h_{k}\right)\right\}_{k \in \mathbb{N}}$ be a $w$-convergent sequence such that

$$
\left(y_{k}, u_{k}, h_{k}\right) \stackrel{w}{\rightarrow}(y, u, h), \quad\left(y_{k}, u_{k}, h_{k}\right) \in \Xi_{\varepsilon_{k}} \text { for every } k \in \mathbb{N},
$$

where $\left\{\varepsilon_{k}\right\}$ is a subsequence of $E$ converging to zero. Then the following inequalities hold (see [35])

$$
\liminf _{k \rightarrow \infty} \int_{\Omega} u_{k}^{2} \mathrm{~d} \mu_{\varepsilon_{k}} \geq \int_{\Omega} u^{2} \mathrm{~d} x, \quad \liminf _{k \rightarrow \infty} \int_{\Gamma_{1}} h_{k}^{2} \mathrm{~d} \mu_{\varepsilon_{k}}^{S} \geq \int_{\Omega} h^{2} \mathrm{~d} l, \quad \liminf _{k \rightarrow \infty} \int_{\Omega} y_{k}^{2} \mathrm{~d} \mu_{\varepsilon_{k}} \geq \int_{\Omega} y^{2} \mathrm{~d} x .
$$

First of all we note that the property of the weak compactness (2.4) for the sequence of measures $\left\{\mu_{\varepsilon}\right\}$ holds automatically in a wider class of test functions, namely when $\varphi \in \mathrm{C}_{0}^{0}\left(\mathbb{R}^{2}\right)$. Thus,

$$
\lim _{k \rightarrow \infty} \int_{\Omega}\left(z_{d}\right)^{2} \mathrm{~d} \mu_{\varepsilon}=\int_{\Omega}\left(z_{d}\right)^{2} \mathrm{~d} x
$$

where $z_{d} \in \mathrm{C}^{0}(\bar{\Omega})$ by the standing assumptions.

Therefore, using this, (6.16) and the definition of the weak two-scale convergence, it follows that

$$
\liminf _{k \rightarrow \infty} \int_{\Omega}\left(y_{k}-z_{d}\right)^{2} \mathrm{~d} \mu_{\varepsilon_{k}}=\liminf _{k \rightarrow \infty} \int_{\Omega} y_{k}^{2} \mathrm{~d} \mu_{\varepsilon_{k}}-2 \int_{\Omega} y z_{d} \mathrm{~d} x+\int_{\Omega}\left(z_{d}\right)^{2} \mathrm{~d} x \geq \int_{\Omega}\left(y-z_{d}\right)^{2} \mathrm{~d} x .
$$

Thus, summing up (6.16) and (6.17), we get $\liminf _{k \rightarrow \infty} I_{\varepsilon_{k}}\left(y_{k}, u_{k}, h_{k}\right) \geq I_{0}(y, u, h)$, i.e. the property (ii) of Definition 5.3 is valid. 
We now verify the correctness of the reverse inequality (5.7). Let $(y, u, h)$ be any triplet of the limit set $\Xi_{0}$, and let $\delta>0$ be a fixed small value. We construct the $\delta$-realizing sequence $\left\{\left(y_{\varepsilon}, u_{\varepsilon}, h_{\varepsilon}\right) \in \Xi_{\varepsilon}\right\}_{\varepsilon \in E}$ such that $u_{\varepsilon}=\widetilde{u}, h_{\varepsilon}=\widetilde{h}$, and $y_{\varepsilon}=y_{\varepsilon}(\widetilde{u}, \widetilde{h})$ is the corresponding solution of the boundary value problem (3.4)-(3.5) under $u=\widetilde{u}$ and $h=\widetilde{h}$. Here $\widetilde{u}$ and $\widetilde{h}$ are the functions with the following properties:

(1) $\widetilde{u} \in \mathrm{C}^{\infty}(\Omega),|\widetilde{u}| \leq c_{u}$ in $\Omega$, and $\|\widetilde{u}-u\|_{\mathrm{L}^{2}(\Omega)} \leq \delta$;

(2) $\widetilde{h} \in \mathrm{C}^{\infty}\left(\Gamma_{1}\right),|\widetilde{h}| \leq c_{h}$ on $\Gamma_{1}$, and $\|\widetilde{h}-h\|_{\mathrm{L}^{2}\left(\Gamma_{1}\right)} \leq \delta$.

It is clear that in this case we have (see (5.3))

$$
u_{\varepsilon} \rightarrow \widetilde{u} \text { in } \mathrm{L}^{2}\left(\Omega, \mathrm{d} \mu_{\varepsilon}\right), \quad h_{\varepsilon} \rightarrow \widetilde{h} \text { in } \mathrm{L}^{2}\left(\Gamma_{1}, \mathrm{~d} \mu_{\varepsilon}^{S}\right) .
$$

Moreover, as follows from the previous lemma, $y_{\varepsilon} \stackrel{2}{\rightarrow} \widetilde{y}$, where $\widetilde{y}$ is a unique solution of the problem (3.4)(3.6) under $u=\widetilde{u}$ and $h=\widetilde{h}$. Hence $\left(y_{\varepsilon}, u_{\varepsilon}, h_{\varepsilon}\right) \stackrel{w}{\longrightarrow}(\widetilde{y}, \widetilde{u}, \widetilde{h})$. In view of the coerciveness property of the homogenized matrix $A^{\text {hom }}$ we have the standard a priori estimate

$$
\|y-\widetilde{y}\|_{\mathrm{H}^{1}(\Omega)} \leq C\left[\|u-\widetilde{u}\|_{\mathrm{L}^{2}(\Omega)}+\|h-\widetilde{h}\|_{\mathrm{L}^{2}\left(\Gamma_{1}\right)}\right] \leq 2 \delta C .
$$

Thus, $\|(y, u, h)-(\widetilde{y}, \widetilde{u}, \widetilde{h})\|_{\mathbb{Y}} \leq 2 \max \{C, 1\} \delta$, that is, $\left\{\left(y_{\varepsilon}, u_{\varepsilon}, h_{\varepsilon}\right)\right\}_{\varepsilon \in E}$ is the $\delta$-realizing sequence with the required properties.

By the initial construction we have

$$
\limsup _{\varepsilon \rightarrow 0} \int_{\Omega} \widetilde{u}_{\varepsilon}^{2} \mathrm{~d} \mu_{\varepsilon}=\int_{\Omega} u^{2} \mathrm{~d} x, \quad \limsup _{\varepsilon \rightarrow 0} \int_{\Gamma_{1}} \widetilde{h}_{\varepsilon}^{2} \mathrm{~d} \mu_{\varepsilon}^{S}=\int_{\Gamma_{1}} h^{2} \mathrm{~d} l .
$$

In order to obtain the convergence

$$
\limsup _{\varepsilon \rightarrow 0} \int_{\Omega}\left(y_{\varepsilon}-z_{d}\right)^{2} \mathrm{~d} \mu_{\varepsilon}=\int_{\Omega}\left(\widetilde{y}-z_{d}\right)^{2} \mathrm{~d} x
$$

we make use the idea of Cioranescu, Murat and Zhikov (see [9,35]). For this we introduce the following auxiliary problem: find $p_{\varepsilon} \in V\left(\Omega, \Gamma_{2}, \mathrm{~d} \mu_{\varepsilon}\right)$ such that

$$
\int_{\Omega}\left[\left(A_{\varepsilon} \nabla p_{\varepsilon}, \nabla \Psi\right)+\alpha p_{\varepsilon} \Psi\right] \mathrm{d} \mu_{\varepsilon}=\int_{\Omega} y_{\varepsilon} \Psi \mathrm{d} \mu_{\varepsilon}, \forall \Psi \in \mathrm{C}^{\infty}\left(\Omega, \Gamma_{2}\right) .
$$

Note that the linear span of the test functions $\Psi \in \mathrm{C}^{\infty}\left(\Omega, \Gamma_{2}\right)$ is dense in $\mathrm{L}^{2}(\Omega)$, hence we may take $p_{\varepsilon}$ as test function in (3.5) under $u=u_{\varepsilon}$ and $h=h_{\varepsilon}$ and $y_{\varepsilon}$ as test function in (6.21). Then, using the symmetry-property of the matrix $A_{\varepsilon}$ we get

$$
\int_{\Omega} y_{\varepsilon}^{2} \mathrm{~d} \mu_{\varepsilon}=\int_{\Omega} u_{\varepsilon} p_{\varepsilon} \mathrm{d} \mu_{\varepsilon}+\int_{\Gamma_{1}} h_{\varepsilon} p_{\varepsilon} \mathrm{d} \mu_{\varepsilon}^{S} \quad \forall \varepsilon \in E .
$$

Since $u_{\varepsilon}=\widetilde{u} \in \mathrm{C}^{\infty}(\Omega), h_{\varepsilon}=\widetilde{h} \in \mathrm{C}^{\infty}\left(\Gamma_{1}\right)$, and $p_{\varepsilon} \rightarrow p$ in $\mathrm{L}^{2}\left(\Omega, \mathrm{d} \mu_{\varepsilon}\right)$, it follows that

$$
\lim _{\varepsilon \rightarrow 0} \int_{\Omega} u_{\varepsilon} p_{\varepsilon} \mathrm{d} \mu_{\varepsilon}=\lim _{\varepsilon \rightarrow 0} \int_{\Omega} \tilde{u} p_{\varepsilon} \mathrm{d} \mu_{\varepsilon}=\int_{\Omega} \tilde{u} p \mathrm{~d} x, \quad \lim _{\varepsilon \rightarrow 0} \int_{\Gamma_{1}} h_{\varepsilon} p_{\varepsilon} \mathrm{d} \mu_{\varepsilon}^{S}=\int_{\Gamma_{1}} \widetilde{h} p \mathrm{~d} l,
$$


where $p \in \mathrm{H}^{1}(\Omega)$ is the solution of a limit problem to (6.21). Note that since $y_{\varepsilon} \stackrel{2}{\rightarrow} \widetilde{y}(x)$ it follows that $p_{\varepsilon} \stackrel{2}{\rightarrow} p(x)$, where

$$
\int_{\Omega}\left[\left(A^{\operatorname{hom}}(x) \nabla p, \nabla \Psi\right)+\alpha p \Psi\right] \mathrm{d} x=\int_{\Omega} \widetilde{y} \Psi \mathrm{d} x, \quad \forall \Psi \in \mathrm{C}^{\infty}\left(\Omega, \Gamma_{2}\right) .
$$

Hence, returning to (6.22), we have

$$
\lim _{\varepsilon \rightarrow 0} \int_{\Omega} y_{\varepsilon}^{2} \mathrm{~d} \mu_{\varepsilon}=\lim _{\varepsilon \rightarrow 0}\left[\int_{\Omega} u_{\varepsilon} p_{\varepsilon} \mathrm{d} \mu_{\varepsilon}+\int_{\Gamma_{1}} h_{\varepsilon} p_{\varepsilon} \mathrm{d} \mu_{\varepsilon}^{S}\right]=\int_{\Omega} \widetilde{u} p \mathrm{~d} x+\int_{\Gamma_{1}} \widetilde{h} p \mathrm{~d} l=\int_{\Omega} \widetilde{y}^{2} \mathrm{~d} x,
$$

i.e. $y_{\varepsilon} \rightarrow \widetilde{y}$ strongly in $\mathrm{L}^{2}\left(\Omega, \mathrm{d} \mu_{\varepsilon}\right)$. Using this fact we immediately obtain

$$
\lim _{\varepsilon \rightarrow 0} \int_{\Omega}\left(y_{\varepsilon}-z_{d}\right)^{2} \mathrm{~d} \mu_{\varepsilon}=\int_{\Omega}\left(\widetilde{y}-z_{d}\right)^{2} \mathrm{~d} x .
$$

As a result, combining (6.19) and (6.23) we deduce $\limsup _{\varepsilon \rightarrow 0} I_{\varepsilon}\left(y_{\varepsilon}, u_{\varepsilon}, h_{\varepsilon}\right)=I_{0}(\widetilde{y}, \widetilde{u}, \widetilde{h})$. Since

$$
\left|I_{0}(\widetilde{y}, \widetilde{u}, \widetilde{h})-I_{0}(y, u, h)\right| \leq \widehat{C} \delta
$$

with some constant $\widehat{C}$ independent of $\delta$, this concludes the proof.

Remark 6.5. Note that the result of the previous lemma remains correct without assuming $A_{\varepsilon}$ to be symmetric, if we assume that the homogenized matrix in Definition 6.1 satisfies the condition $A_{\varepsilon}^{T}(x) \nabla y_{\varepsilon} \rightarrow\left(A^{\text {hom }}\right)^{T} \nabla y_{0}$ in $L^{2}\left(\Omega, \mathrm{d} \mu_{\varepsilon}\right)^{2}$ for every $\left\{y_{\varepsilon}\right\}$ such that

$$
\begin{array}{r}
\left\{(y, u, h) \in \mathbb{Z}_{\varepsilon}\left(\Omega, \Gamma_{1}\right): \int_{\Omega}\left[\left(A_{\varepsilon}^{T}(x) \nabla y, \nabla \varphi\right)+\alpha y \varphi\right] \mathrm{d} \mu_{\varepsilon}=\int_{\Omega} u \varphi \mathrm{d} \mu_{\varepsilon}+\int_{\Gamma_{1}} h \varphi \mathrm{d} \mu_{\varepsilon}^{S}, \forall \varphi \in \mathrm{C}^{\infty}\left(\Omega, \Gamma_{2}\right)\right\} \\
\ni\left(y_{\varepsilon}, u_{\varepsilon}, h_{\varepsilon}\right) \rightarrow\left(y_{0}, u_{0}, h_{0}\right) \text { in the sense of } w \text {-convergence. }
\end{array}
$$

Furthermore, we emphasize that this property holds automatically for a $\mu$-measurable symmetric periodic matrix $A_{\varepsilon}(x)=A\left(\varepsilon^{-1} x\right)$ satisfying the condition of ellipticity and boundedness (3.2).

Now we are in a position to prove the main result of this section.

Theorem 6.6. Under supposition of Lemma 6.2 for the family of problems (3.3)-(3.6) there exists a unique homogenized optimal control problem which has the following representation

$$
\begin{gathered}
-\operatorname{div}\left(A^{\mathrm{hom}}(x) \nabla y\right)+\alpha y=u \quad \text { in } \Omega, \\
y=0 \quad \text { on } \Gamma_{2} \quad \partial y / \partial \nu_{A^{\mathrm{hom}}}=h \quad \text { on } \Gamma_{1}, \\
|u| \leq c_{u} \quad \text { a.e. in } \Omega, \quad|h| \leq c_{h} \quad \text { a.e. on } \Gamma_{1}, \\
I_{0}(y, u, h)=k_{1} \int_{\Omega}\left(y-z_{d}\right)^{2} \mathrm{~d} x+k_{2} \int_{\Omega} u^{2} \mathrm{~d} x+k_{3} \int_{\Gamma_{1}} h^{2} \mathrm{~d} l \rightarrow \mathrm{inf},
\end{gathered}
$$

where $\partial y / \partial \nu_{A^{\text {hom }}}=\sum_{i, j=1}^{2} a_{i j}^{\text {hom }}(x) \frac{\partial y}{\partial x_{i j}} \cos \left(n, x_{i}\right), \cos \left(n, x_{i}\right)$ is $i$-th direction cosine of $n, n$ being the normal at $\Gamma_{1}$ exterior to $\Omega$. 
Moreover, the sequence of optimal solutions $\left\{\left(y_{\varepsilon}^{0}, u_{\varepsilon}^{0}, h_{\varepsilon}^{0}\right) \in \Xi_{\varepsilon}\right\}$ for the original problems (3.3)-(3.6) and corresponding minimal values of the cost functional (3.3) satisfy the following variational properties:

$$
\begin{gathered}
\lim _{\varepsilon \rightarrow 0} \inf _{(y, u, h) \in \Xi_{\varepsilon}} I_{\varepsilon}(y, u, h)=I_{0}\left(y^{0}, u^{0}, h^{0}\right)=\inf _{(y, u, h) \in \Xi_{0}} I_{0}(y, u, h), \\
y_{\varepsilon}^{0} \stackrel{2}{\rightarrow} y^{0}, \quad u_{\varepsilon}^{0} \rightarrow u^{0} \text { in } \mathrm{L}^{2}\left(\Omega, \mathrm{d} \mu_{\varepsilon}\right), \quad h_{\varepsilon}^{0} \rightarrow h^{0} \text { in } \mathrm{L}^{2}\left(\Gamma_{1}, \mathrm{~d} \mu_{\varepsilon}^{S}\right),
\end{gathered}
$$

where $\left(y^{0}, u^{0}, h^{0}\right)$ is a unique solution of the homogenized problem (6.24)-(6.27).

Proof. As immediately follows from Lemmas 3.7 and 6.2 for the sequence of constrained minimization problems (3.11) there exists a variational limit (3.12) the main components of which can be recovered in the form (6.2) and (6.14), respectively. Moreover, from (6.2) we have the following implication

$$
(y, u, h) \in \Xi_{0} \Rightarrow(y, u, h) \in \operatorname{Graph}\left(\mathbb{P}^{\text {hom }}\right) .
$$

It is clear now that the limit problem (3.12) can be written in the form of the optimal control problem (6.24)(6.27). Therefore, in view of Definition 3.8 the problem (6.24)-(6.27) is the homogenized optimal control problem for the original family (3.3)-(3.6).

Applying now Theorem 5.7 we come to the following variational properties of the homogenized problem (6.24)-(6.27): let $\left\{\left(y_{\varepsilon}^{0}, u_{\varepsilon}^{0}, h_{\varepsilon}^{0}\right)\right\}$ be the sequence of optimal triplets for the problems (3.3)-(3.6), then

$$
\begin{gathered}
\lim _{\varepsilon \rightarrow 0} I_{\varepsilon}\left(y_{\varepsilon}^{0}, u_{\varepsilon}^{0}, h_{\varepsilon}^{0}\right)=\lim _{\varepsilon \rightarrow 0} \inf _{\left(y_{\varepsilon}, u_{\varepsilon}, h_{\varepsilon}\right) \in \Xi_{\varepsilon}} I_{\varepsilon}\left(y_{\varepsilon}, u_{\varepsilon}, h_{\varepsilon}\right)=\inf _{(y, u, h) \in \Xi_{0}} I_{0}(y, u, h)=I_{0}\left(y^{0}, u^{0}, h^{0}\right), \\
\left(y_{\varepsilon}^{0}, u_{\varepsilon}^{0}, h_{\varepsilon}^{0}\right) \stackrel{w}{\longrightarrow}\left(y^{0}, u^{0}, h^{0}\right) .
\end{gathered}
$$

Hence $I_{\varepsilon}\left(y_{\varepsilon}^{0}, u_{\varepsilon}^{0}, h_{\varepsilon}^{0}\right) \rightarrow I_{0}\left(y^{0}, u^{0}, h^{0}\right)$. Rewriting this in the explicit form we get

$$
\begin{aligned}
\lim _{\varepsilon \rightarrow 0}\left[k_{1} \int_{\Omega}\left(y_{\varepsilon}^{0}-z_{d}\right)^{2} \mathrm{~d} \mu_{\varepsilon}+k_{2} \int_{\Omega}\left(u_{\varepsilon}^{0}\right)^{2} \mathrm{~d} \mu_{\varepsilon}+k_{3} \int_{\Gamma_{1}}\left(h_{\varepsilon}^{0}\right)^{2} \mathrm{~d} \mu_{\varepsilon}^{S}\right] & = \\
& k_{1} \int_{\Omega}\left(y^{0}-z_{d}\right)^{2} \mathrm{~d} x+k_{2} \int_{\Omega}\left(u^{0}\right)^{2} \mathrm{~d} x+k_{3} \int_{\Gamma_{1}}\left(h^{0}\right)^{2} \mathrm{~d} l .
\end{aligned}
$$

Therefore the validity of this equality for every $k_{i} \geq 0$ implies

$$
\lim _{\varepsilon \rightarrow 0} \int_{\Omega}\left(y_{\varepsilon}^{0}\right)^{2} \mathrm{~d} \mu_{\varepsilon}=\int_{\Omega}\left(y^{0}\right)^{2} \mathrm{~d} x, \quad \lim _{\varepsilon \rightarrow 0} \int_{\Omega}\left(u_{\varepsilon}^{0}\right)^{2} \mathrm{~d} \mu_{\varepsilon}=\int_{\Omega}\left(u^{0}\right)^{2} \mathrm{~d} x, \quad \lim _{\varepsilon \rightarrow 0} \int_{\Gamma_{1}}\left(h_{\varepsilon}^{0}\right)^{2} \mathrm{~d} \mu_{\varepsilon}^{S}=\int_{\Omega}\left(h^{0}\right)^{2} \mathrm{~d} l .
$$

Combining these properties with (6.30) and using Proposition 4.1 we obtain the required assertions (6.28)-(6.29). This concludes the proof.

\section{Construction of suboptimal controls}

We now focus on the construction of approximations to the optimal solution of the original problem (3.3)(3.6) for $\varepsilon$ small enough. We define suboptimal controls $\left(u_{\varepsilon}^{\text {sub }}, h_{\varepsilon}^{\text {sub }}\right)$ which approximate the optimal value of the original problem (3.3)-(3.6). To do so, we introduce the following concept: 
Definition 7.1. We say that a sequence of pairs $\left\{\left(u_{\varepsilon}^{\text {sub }}, h_{\varepsilon}^{\text {sub }}\right)\right\}_{\varepsilon>0}$ is asymptotically suboptimal to the problem (3.3)-(3.6) if there exists a constant $\widehat{C}>0$ independent of $\varepsilon$ such that for every $\delta\left(0<\delta<\delta_{0}\right)$ there is $\varepsilon_{0}>0$ satisfying

$$
\left|\inf _{(y, u, h) \in \Xi_{\varepsilon}} I_{\varepsilon}(y, u, h)-I_{\varepsilon}\left(y_{\varepsilon}^{\mathrm{sub}}, u_{\varepsilon}^{\mathrm{sub}}, h_{\varepsilon}^{\mathrm{sub}}\right)\right|<\widehat{C} \delta \quad \forall \varepsilon<\varepsilon_{0}
$$

where $y_{\varepsilon}^{\mathrm{sub}}=y_{\varepsilon}^{\mathrm{sub}}\left(u_{\varepsilon}^{\mathrm{sub}}, h_{\varepsilon}^{\mathrm{sub}}\right)$ denote the corresponding solutions of the boundary value problem (3.4)-(3.5).

Theorem 6.6 leads to the following final result:

Theorem 7.2. Let $u^{0} \in \mathrm{L}^{2}(\Omega)$ and $h^{0} \in \mathrm{L}^{2}\left(\Gamma_{1}\right)$ be the optimal controls for the homogenized problem (6.24)(6.27). Then any $\delta$-realizing sequence to the pair $\left(u^{0}, h^{0}\right)$ is asymptotically suboptimal for the original optimal control problem (3.3)-(3.6).

Proof. Let $\left(y^{0}, u^{0}, h^{0}\right)$ be a unique solution of the homogenized problem (6.24)-(6.27). For a given $\delta>0$ we construct the $\delta$-realizing sequence $\left\{\left(y_{\varepsilon}, u_{\varepsilon}, h_{\varepsilon}\right) \in \Xi_{\varepsilon}\right\}_{\varepsilon \in E}$ by the usual way, that is, $u_{\varepsilon}=\widetilde{u}, h_{\varepsilon}=\widetilde{h}$, and $y_{\varepsilon}=y_{\varepsilon}(\widetilde{u}, \widetilde{h})$ is the corresponding solution of the boundary value problem (3.4)-(3.5) under $u=\widetilde{u}$ and $h=\widetilde{h}$. Here

$$
\left.\begin{array}{lll}
\widetilde{u} \in \mathrm{C}^{\infty}(\Omega), & |\widetilde{u}| \leq c_{u} \quad \text { in } \Omega, \quad\left\|\widetilde{u}-u^{0}\right\|_{\mathrm{L}^{2}(\Omega)} \leq \delta, \\
\widetilde{h} \in \mathrm{C}^{\infty}\left(\Gamma_{1}\right), & |\widetilde{h}| \leq c_{h} \quad \text { on } \Gamma_{1}, \quad\left\|\widetilde{h}-h^{0}\right\|_{\mathrm{L}^{2}\left(\Gamma_{1}\right)} \leq \delta .
\end{array}\right\}
$$

It is clear that (see Lem. 6.4)

$$
\begin{gathered}
\limsup _{\varepsilon \rightarrow 0} \int_{\Omega}\left(y_{\varepsilon}-z_{d}\right)^{2} \mathrm{~d} \mu_{\varepsilon}=\int_{\Omega}\left(\widetilde{y}-z_{d}\right)^{2} \mathrm{~d} x, \quad\left\|y^{0}-\widetilde{y}\right\|_{\mathrm{H}^{1}(\Omega)} \leq 2 \delta C(\text { see (6.18)), } \\
\limsup _{\varepsilon \rightarrow 0} \int_{\Omega} \widetilde{u}_{\varepsilon}^{2} \mathrm{~d} \mu_{\varepsilon}=\int_{\Omega}\left(u^{0}\right)^{2} \mathrm{~d} x, \quad \limsup _{\varepsilon \rightarrow 0} \int_{\Gamma_{1}} \widetilde{h}_{\varepsilon}^{2} \mathrm{~d} \mu_{\varepsilon}^{S}=\int_{\Gamma_{1}}\left(h^{0}\right)^{2} \mathrm{~d} l,
\end{gathered}
$$

where $\widetilde{y}$ is a unique solution of the problem (6.24)-(6.25) under $u=\widetilde{u}$ and $h=\widetilde{h}$. Besides, in view of these relations, we have the following obvious estimates:

$$
\begin{aligned}
k_{2}\left|\int_{\Omega}\left(u^{0}\right)^{2} \mathrm{~d} x-\int_{\Omega}(\widetilde{u})^{2} \mathrm{~d} x\right| & \leq k_{2}\left(\delta_{0}+2\left\|u^{0}\right\|_{\mathrm{L}^{2}(\Omega)}\right) \delta=C_{2} \delta, \\
k_{3}\left|\int_{\Omega}\left(h^{0}\right)^{2} \mathrm{~d} x-\int_{\Omega}(\widetilde{h})^{2} \mathrm{~d} x\right| & \leq k_{3}\left(\delta_{0}+2\left\|h^{0}\right\|_{\mathrm{L}^{2}\left(\Gamma_{1}\right)}\right) \delta=C_{3} \delta, \\
k_{1}\left|\int_{\Omega}\left(y^{0}-z_{d}\right)^{2} \mathrm{~d} x-\int_{\Omega}\left(\widetilde{y}-z_{d}\right)^{2} \mathrm{~d} x\right| & \leq 4 k_{1}\left(\delta_{0} C+\left\|y^{0}-z_{d}\right\|_{\mathrm{L}^{2}(\Omega)}\right) C \delta=C_{1} \delta .
\end{aligned}
$$




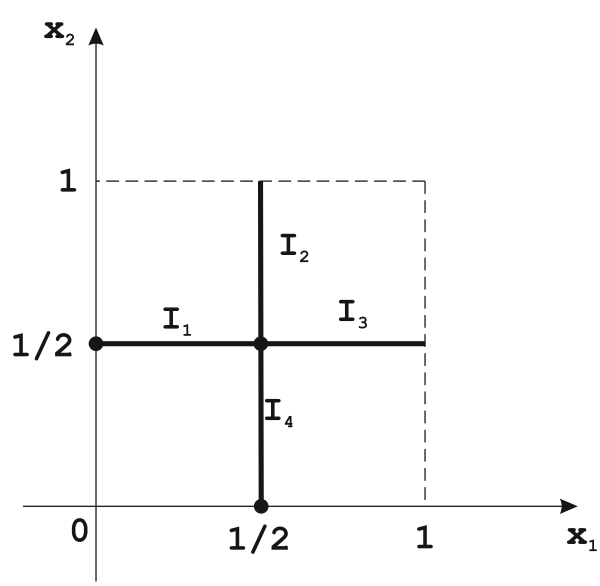

(a)

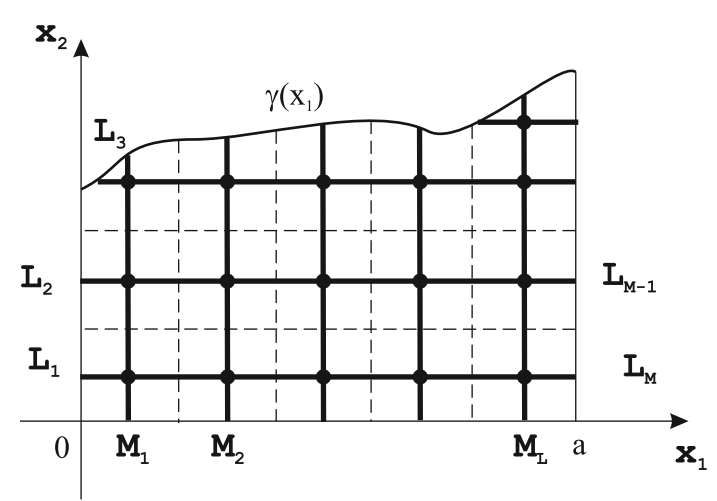

(b)

Figure 2. (a) The cell of grid periodicity. (b) Periodic grid on $\Omega$.

We now observe that

$$
\begin{aligned}
& \left|\inf _{(y, u, h) \in \Xi_{\varepsilon}} I_{\varepsilon}(y, u, h)-I_{\varepsilon}\left(y_{\varepsilon}, u_{\varepsilon}, h_{\varepsilon}\right)\right|=\left|I_{\varepsilon}\left(y_{\varepsilon}^{0}, u_{\varepsilon}^{0}, h_{\varepsilon}^{0}\right)-I_{\varepsilon}\left(y_{\varepsilon}, \widetilde{u}, \widetilde{h}\right)\right| \leq\left|I_{\varepsilon}\left(y_{\varepsilon}^{0}, u_{\varepsilon}^{0}, h_{\varepsilon}^{0}\right)-I_{0}\left(y^{0}, u^{0}, h^{0}\right)\right| \\
& +\left|I_{0}(\widetilde{y}, \widetilde{u}, \widetilde{h})-I_{\varepsilon}\left(y_{\varepsilon}, \widetilde{u}, \widetilde{h}\right)\right|+\left|I_{0}\left(y^{0}, u^{0}, h^{0}\right)-I_{0}(\widetilde{y}, \widetilde{u}, \widetilde{h})\right| \leq\left|I_{\varepsilon}\left(y_{\varepsilon}^{0}, u_{\varepsilon}^{0}, h_{\varepsilon}^{0}\right)-I_{0}\left(y^{0}, u^{0}, h^{0}\right)\right| \\
& +\left|I_{0}(\widetilde{y}, \widetilde{u}, \widetilde{h})-I_{\varepsilon}\left(y_{\varepsilon}, \widetilde{u}, \widetilde{h}\right)\right|+k_{1}\left|\int_{\Omega}\left(y^{0}-z_{d}\right)^{2} \mathrm{~d} x-\iint_{\Omega}\left(\widetilde{y}-z_{d}\right)^{2} \mathrm{~d} x\right| \\
& +k_{2}\left|\int_{\Omega}\left(u^{0}\right)^{2} \mathrm{~d} x-\int_{\Omega}(\widetilde{u})^{2} \mathrm{~d} x\right|+k_{3}\left|\int_{\Gamma_{1}}\left(h^{0}\right)^{2} \mathrm{~d} l-\int_{\Gamma_{1}}(\widetilde{h})^{2} \mathrm{~d} l\right|=J_{1}+J_{2}+J_{3}+J_{4}+J_{5} .
\end{aligned}
$$

To conclude the proof, we note that for a given $\delta>0$ one can always find: (1) $\varepsilon_{1}>0$ such that $J_{1}<\delta / 2$ for all $\varepsilon<\varepsilon_{1}$ by Theorem 6.6 ; (2) $\varepsilon_{2}>0$ such that $J_{2}<\delta / 2$ for all $\varepsilon<\varepsilon_{2}$ by Lemma 6.4. Besides, $J_{3}<C_{1} \delta$, $J_{4}<C_{2} \delta$, and $J_{5}<C_{3} \delta$ by estimates (7.5)-(7.7). As a result, we have

$$
\left|\inf _{(y, u, h) \in \Xi_{\varepsilon}} I_{\varepsilon}(y, u, h)-I_{\varepsilon}\left(y_{\varepsilon}, u_{\varepsilon}, h_{\varepsilon}\right)\right| \leq\left(1+C_{1}+C_{2}+C_{3}\right) \delta
$$

for all $\varepsilon<\min \left\{\varepsilon_{1}, \varepsilon_{2}\right\}$. Thus, we have obtained the required estimate (7.1).

\section{An EXAMPle FOR THE HOMOGENizATION OF AN OPTIMAL CONTROL PROBLEM ON AN $\varepsilon$-PERIODIC SQUARE GRID}

On the domain $\Omega$ that was defined in $(2.1)$ we consider the $\varepsilon$-periodic square grid $\varepsilon \mathcal{F}$ with the cell of periodicity $\varepsilon \square$. Here the set $\square=[0,1)^{2}$ contains the "cross"-structure such as indicated in Figure 2(a).

Following the notation of Section 1 we say that $\Omega_{\varepsilon}$ has $\varepsilon$-periodic grid-like structure if $\Omega_{\varepsilon}=\Omega \cap \varepsilon \mathcal{F}$. As usual we set $\partial \Omega=\Gamma_{1} \cup \Gamma_{2}$, where $\Gamma_{1}=\left\{x \in \bar{\Omega} \mid x_{2}=0,0<x_{1}<a\right\}$.

We begin with some standard notations on graphs (see [22]). Let $V^{\varepsilon}=\left\{v_{J}: J \in \mathcal{J}_{\varepsilon}\right\}$ be the set of vertices of our $\varepsilon$-periodic graph (grid) $\Omega_{\varepsilon}$, let $E^{\varepsilon}=\left\{e_{i}: i \in \dot{\mathcal{I}}_{\varepsilon}\right\}$ be the index set of corresponding edges. Here by $\mathcal{J}_{\varepsilon}$ 
and $\dot{\mathcal{I}}_{\varepsilon}$ we denote the index sets for vertices and edges, respectively. For given vertex $v_{J}$ we consider the set of edges that are incident at $v_{J}$. The corresponding set of edge-indices is denoted by

$$
\dot{\mathcal{I}}_{J}=\left\{i \in \dot{\mathcal{I}}_{\varepsilon}: e_{i} \text { is incident at } v_{J}\right\} .
$$

The cardinality of $\dot{\mathcal{I}}_{J}$ is the edge degree at $v_{J}$ that is $d_{J}=\left|\dot{\mathcal{I}}_{J}\right|$. It is easy to see that $d_{J} \leq 4$ in our case.

Note that as follows from Figure 2(b) every edge $e_{i}$ on the graph $\Omega_{\varepsilon}$ can be parameterized by $x \in\left[0, l_{i}\right]$ where $l_{i} \leq \varepsilon / 2$ denotes the length of the edge $e_{i}$. With every edge $e_{i}$ we will associate a so-called "state-function"

$$
y_{i}:\left[0, l_{i}\right] \rightarrow \mathbb{R}^{1}, i \in \dot{\mathcal{I}}_{\varepsilon} .
$$

Since we are going to consider an optimal control problem on bounded periodic graph it is necessary to specify boundary and so-called transmission conditions at the vertices $V^{\varepsilon}$ of $\Omega_{\varepsilon}$. To this end we subdivide the set of vertices (nodes) as follows:

$$
V^{\varepsilon}=V_{S}^{\varepsilon} \cup V_{M}^{\varepsilon},
$$

where $V_{S}^{\varepsilon}$ denotes the set of simple modes such that $d_{J}=1$ and $V_{M}^{\varepsilon}$ signifies the set of multiple nodes where $4 \geq d_{J}>1$. The set of simple nodes $V_{S}^{\varepsilon}$ will be divided as

$$
V^{\varepsilon}=V_{\Gamma_{1}}^{\varepsilon} \cup V_{\Gamma_{2}}^{\varepsilon}
$$

where $V_{\Gamma_{1}}^{\varepsilon}$ represent the set of simple nodes belonging to $\Gamma_{1}$-boundary and $V_{\Gamma_{2}}^{\varepsilon}$ signifies those simple nodes which are belonging to $\Gamma_{2}$-boundary. It is easy to see that in the case of $\varepsilon$-periodic grid on $\Omega$ there is not any simple node lying in the interior of the domain $\Omega$. Further we will look at the set $V_{\Gamma_{1}}^{\varepsilon}$ as the set of control-active Neumann nodes and at $V_{\Gamma_{2}}^{\varepsilon}$ as the set of nodes with zero Dirichlet conditions.

On all edges $e_{i}$ we consider differential operator $L_{i}$ of the following form

$$
L_{i} y_{i}=-R_{i} y_{i}^{\prime \prime}+\alpha y_{i}
$$

where $R_{i} \geq \alpha>0$. Moreover, using the $\varepsilon$-periodic structure of $\Omega_{\varepsilon}$ we will always suppose that for every $\varepsilon$-cell $\varepsilon \square_{j}$ we have (see Fig. 4(a))

where $\alpha^{-1} \geq \beta, \gamma \geq \alpha>0$.

$$
R_{i}=\beta, \quad R_{i+1}=\gamma, \quad R_{i+2}=\beta, \quad R_{i+3}=\gamma,
$$

We now define the classes of admissible controls $U^{\varepsilon}$ and $H^{\varepsilon}$ where

$$
\begin{aligned}
& U^{\varepsilon}=\left\{u: \Omega_{\varepsilon} \rightarrow \mathbb{R}^{1}:\left.u\right|_{l_{i}} \in \mathrm{L}^{2}\left(0, l_{i}\right) ; \quad|u(x)| \leq c_{u} \quad \text { for almost every } x \in \Omega_{\varepsilon}\right\}, \\
& H^{\varepsilon}=\left\{h=\left(h_{1}, h_{2}, \ldots, h_{L_{\varepsilon}}\right) \in \mathbb{R}^{L_{\varepsilon}}: L_{\varepsilon}=\left|V_{\Gamma_{1}}^{\varepsilon}\right|, \quad \sum_{K=1}^{L_{\varepsilon}} h_{K}^{2}<\infty, \quad\left|h_{K}\right| \leq c_{h}\right\} .
\end{aligned}
$$

Here $c_{u}, c_{h}$ are some positive constant, by $\left|V_{\Gamma_{1}}^{\varepsilon}\right|$ we denote the amount of all simple nodes belonging to $\Gamma_{1}$.

Let $k_{1}, k_{2}, k_{3}\left(k_{i}>0\right)$ be penalty terms and let $z_{d}, u_{d}$, and $h_{d}$ be given functions of $\mathrm{C}^{0}(\bar{\Omega})$. We consider the following optimal control problem on the grid $\Omega_{\varepsilon}$

$$
\begin{gathered}
-R_{i} y_{i}^{\prime \prime}+\alpha y_{i}=u_{i}, \quad x \in\left(0, l_{i}\right), \quad i \in \dot{\mathcal{I}}_{\varepsilon}, \\
y_{i}\left(v_{J}\right)=0, \quad i \in \dot{\mathcal{I}}_{J}^{\varepsilon}, \quad v_{J} \in V_{\Gamma_{2}}^{\varepsilon}, \\
\sum_{i \in \mathcal{I}_{J}^{\varepsilon}} R_{i} y_{i}^{\prime}\left(v_{J}\right)=0, \quad v_{J} \in V_{M}^{\varepsilon}, \\
y_{i}\left(v_{J}\right)=h_{k(J)}, \quad \forall v_{J} \in V_{\Gamma_{1}}^{\varepsilon}, \quad i \in \mathcal{I}_{J}^{\varepsilon} ; \quad k(J) \in\left\{1,2, \ldots, L_{\varepsilon}\right\},
\end{gathered}
$$




$$
\begin{gathered}
u \in U^{\varepsilon}, \quad h \in H^{\varepsilon}, \\
I_{\varepsilon}(y, u, h)=\sum_{i \in \dot{I}_{\varepsilon}} k_{1} \int_{0}^{l_{i}}\left(y_{i}-\left.z_{d}\right|_{e_{i}}\right)^{2} \mathrm{~d} x+k_{2} \sum_{i \in \dot{\mathcal{I}}_{\varepsilon}} \int_{0}^{l_{i}}\left(u_{i}-\left.u_{d}\right|_{e_{i}}\right)^{2} \mathrm{~d} x+k_{3} \sum_{K=1}^{L_{\varepsilon}}\left(h_{K}-h_{d}\left(v_{J}\right)\right)^{2} \rightarrow \inf .
\end{gathered}
$$

Using the property of the sets $U^{\varepsilon}$ and $H^{\varepsilon}$ and invoking the standard arguments it is easy to prove that for every $\varepsilon \in E$ the problem (8.4)-(8.9) admits a unique optimal triplet $\left(y_{\varepsilon}^{0}, u_{\varepsilon}^{0}, h_{\varepsilon}^{0}\right)$ which can be characterized by some adjoint system.

Our aim is to study the asymptotic behavior of this problem as $\varepsilon$ tends to 0 . For this we reformulate the problem (8.4)-(8.9) in the terms of some variational control problem defined on spaces with singular measures. We introduce the $\square$-periodic Borel measure $\mu$ in $\mathbb{R}^{2}$ as follows:

$$
\mu=\frac{1}{2}\left(\mu_{1}+\mu_{2}+\mu_{3}+\mu_{4}\right)
$$

where $\mu_{i}$ are the 1-dimensional Lebesgue measures on the corresponding line segments (edges) $I_{i}$ (see Fig. 2).

Also we define the $\square^{S}$-periodic Radon measure $\mu^{S}$ in $\mathbb{R}^{1}$ as $\mu^{S}=\delta_{(1 / 2,0)}$, where $\delta_{(1 / 2,0)}$ is Dirac measure at the point $(1 / 2,0) \in \square^{S}$. It is easy to see that

$$
\int_{\square} \mathrm{d} \mu=1 \text { and } \int_{\square^{S}} \mathrm{~d} \mu^{S}=1 .
$$

Therefore we may define the "scaling" measures

$$
\mu_{\varepsilon}(B)=\varepsilon^{2} \mu\left(\varepsilon^{-1} B\right), \quad \mu_{\varepsilon}^{S}\left(B_{1}\right)=\varepsilon \mu^{S}\left(\varepsilon^{-1} B_{1}\right),
$$

where $B, B_{1}$ are corresponding Borel sets in $\mathbb{R}^{2}$ and $\mathbb{R}^{1}$ respectively. Obviously, each of these measures $\mu_{\varepsilon}$, $\mu_{\varepsilon}^{S}$ converges weakly to the corresponding Lebesgue measure: $\mathrm{d} \mu_{\varepsilon} \rightarrow \mathrm{d} x, \mathrm{~d} \mu_{\varepsilon}^{S} \rightarrow \mathrm{d} l$. Here $\mathrm{d} x$ and $\mathrm{d} l$ are the Lebesgue measures in $\mathbb{R}^{2}$ and $\mathbb{R}^{1}$, respectively.

Now we define the matrix $A_{\varepsilon}(x)=A\left(\varepsilon^{-1} x\right)$ as follows

$$
A(z)=\left[\begin{array}{ll}
a_{11}(z) & 0 \\
0 & a_{22}(z)
\end{array}\right]
$$

where $a_{11}(z)=\beta$ and $a_{22}(z)=\gamma$. It is easy to see that such defined matrix is symmetric, $\mu$-measurable and satisfying the property (3.2).

As a result the original optimal control problem can be presented in the form

$$
\begin{gathered}
\int_{\Omega}\left[\left(A\left(\varepsilon^{-1} x\right) \nabla y, \nabla \varphi\right)+\alpha y \varphi\right] \mathrm{d} \mu_{\varepsilon}=\int_{\Omega} u \varphi \mathrm{d} \mu_{\varepsilon}+\int_{\Gamma_{1}} h \varphi \mathrm{d} \mu_{\varepsilon}^{S}, \quad \forall \varphi \in \mathrm{C}^{\infty}\left(\Omega, \Gamma_{2}\right), \\
y \in V\left(\Omega, \Gamma_{2}, \mathrm{~d} \mu_{\varepsilon}\right), \quad|u| \leq c_{u} \quad \mu_{\varepsilon} \text {-a.e. in } \Omega, \quad|h| \leq c_{h} \quad \mu_{\varepsilon}^{S} \text {-a.e. on } \Gamma_{1}, \\
I_{\varepsilon}(y, u, h)=k_{1} \int_{\Omega}\left(y-z_{d}\right)^{2} \mathrm{~d} \mu_{\varepsilon}+k_{2} \int_{\Omega}\left(u-u_{d}\right)^{2} \mathrm{~d} \mu_{\varepsilon}+k_{3} \int_{\Gamma_{1}}\left(h-h_{d}\right)^{2} \mathrm{~d} \mu_{\varepsilon}^{S} \rightarrow \inf .
\end{gathered}
$$

The validity of this representation immediately follows from the Proposition 3.2 and Remark 4.4. Then, due to Theorem 6.6, the control problem (8.10)-(8.12) admits a homogenization and the limit problem can be 


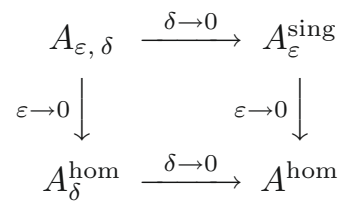

FiguRE 3. Homogenization diagram.

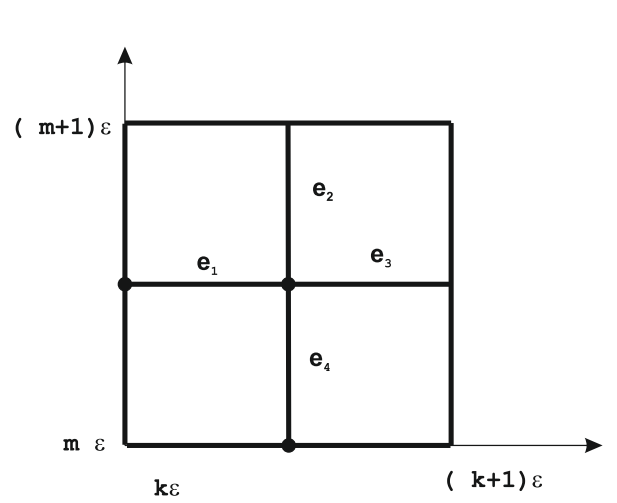

(a)

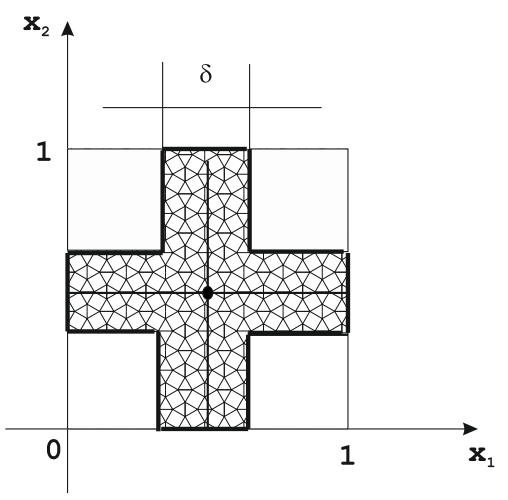

(b)

Figure 4. (a) $\varepsilon \square_{J}$-cell. (b) Periodicity cell for $\mathcal{F}^{\delta}$.

recovered in the form

$$
\begin{gathered}
-\operatorname{div}\left(A^{\mathrm{hom}} \nabla y\right)+\alpha y=u \text { in } \Omega, \\
y=0 \text { on } \Gamma_{2}, \quad \partial y / \partial \nu_{A^{\mathrm{hom}}}=h \text { on } \Gamma_{1}, \\
|u| \leq c_{u} \text { a.e. in } \Omega, \quad|h| \leq c_{h} \text { a.e. on } \Gamma_{1}, \\
I_{0}(y, u, h)=k_{1} \int_{\Omega}\left(y-z_{d}\right)^{2} \mathrm{~d} x+k_{2} \int_{\Omega}\left(u-u_{d}\right)^{2} \mathrm{~d} x+k_{3} \int_{\Gamma_{1}}\left(h-h_{d}\right)^{2} \mathrm{~d} l .
\end{gathered}
$$

The identification of the matrix $A^{\text {hom }}$ can be done in different ways either by definition, i.e. as the solution of the minimum problem $\left(A^{\text {hom }} \xi, \xi\right)=\min _{p \in V_{\text {pot }}}(A(\xi+p), \xi+p)$ (see [35]), or using a more classical method: firstly, we homogenize the problem on the grid with non-zero thickness in a usual way (see $[31,37]$ ) and then pass to the limit as thickness goes to zero. As for the second approach it was shown in [8] that in this case the corresponding diagram of homogenization (see Fig. 3) is commutative.

Here $\delta$ denotes a small parameter which characterizes the fixed "thickness" of graphs. The corresponding structures will be called $\delta$-grids $\mathcal{F}^{\delta}$.

We will follow the second approach. Therefore we define the $\varepsilon$-periodic $\delta$-grids $\mathcal{F}_{\varepsilon}^{\delta}$ be setting $\mathcal{F}_{\varepsilon}^{\delta}=\varepsilon^{-1} \mathcal{F}^{\delta}$, the cell of periodicity $\square_{\delta}$ for $\mathcal{F}^{\delta}$ has the form that Figure 4(b) shows.

Let $\mu_{\delta}^{\varepsilon}(B)=\varepsilon^{2} \mu_{\delta}\left(\varepsilon^{-1} B\right)$ and $\mu_{\delta}$ is the measure on $\mathcal{F}^{\delta}$ that can be defined as the probability measure in $\Omega$ supported by the cross-bar in $\square_{\delta}$ and is uniformly distributed on it. The weak limit of this measure $\mu_{\delta}$ as $\delta \rightarrow 0$ is the singular measure $\mu$ which was defined before. Assume that the matrix $A_{\varepsilon, \delta}=A\left(\varepsilon^{-1} x\right)$, i.e. $A_{\varepsilon, \delta}=\operatorname{diag}(\beta, \gamma)$. 
Then using the standard technique of homogenization (see [29,31]) we get $A_{\varepsilon, \delta} \stackrel{\varepsilon \rightarrow 0}{\longrightarrow} A_{\delta}^{\text {hom }}$, where

$$
A_{\delta}^{\text {hom }}=\left[\begin{array}{cc}
b_{11}^{\delta} & 0 \\
0 & b_{22}^{\delta}
\end{array}\right], \quad b_{11}^{\delta}=\frac{1}{(2-\delta)}\left[\beta+o\left(\delta^{1 / 2}\right)\right], \quad b_{22}^{\delta}=\frac{1}{(2-\delta)}\left[\gamma+o\left(\delta^{1 / 2}\right)\right] .
$$

Furthermore, due to the results of Saint Jean Paulin and Cioranescu [31] we may take the limit $A_{\delta}^{\text {hom }} \stackrel{\delta \rightarrow 0}{\longrightarrow}$ $A^{\text {hom }}$. As a result we obtain (for details we refer to $\left.[2,28,29,31]\right) A^{\text {hom }}=\operatorname{diag}\left(\frac{1}{2} \beta, \frac{1}{2} \gamma\right)$.

Thus the homogenized state equation for the limit problem (8.13)-(8.16) has the form

$$
-\beta \frac{\partial^{2} y}{\partial x_{1}^{2}}-\gamma \frac{\partial^{2} y}{\partial x_{2}^{2}}+2 \alpha y=2 u \quad \text { in } \Omega, \quad y=0 \quad \text { on } \quad \Gamma_{2}, \quad \gamma \frac{\partial y}{\partial x_{2}}=2 h \text { on } \Gamma_{1} .
$$

Furthermore, in view of the result of Theorem 6.6 the homogenized optimal control problem (8.13)-(8.16) satisfies the variational properties (6.28)-(6.29). Note also that another approach for the identification of homogenized operators $-\operatorname{div}\left(A^{\text {hom }} \nabla y\right)$, the so called direct asymptotic method, was proposed by Mazja and Slutskij in [26].

In the case when $a=2, \alpha=\beta=\gamma=1, \Omega=\left\{\left(x_{1}, x_{2}\right): x_{1} \in(0,2), 0<x_{2}<\sqrt{2 x_{1}-x_{1}^{2}}\right\}, h_{d}=0$, $z_{d}=\exp (r), u_{d}=\exp (r)\left(1-2 r^{2}-6 r^{3}-2 r^{4}\right), r=\left(x_{1}^{2}+x_{2}^{2}-2 x_{1}\right)^{-1}$, we have that

$$
u^{0}=\exp (r)\left(1-2 r^{2}-6 r^{3}-2 r^{4}\right) \quad \text { and } \quad h^{0}=0
$$

are the optimal controls to the homogenized problem (8.13)-(8.16). Hence, in view of Theorem 6.6 the restriction of these functions on the sets $\Omega_{\varepsilon}$ and $V_{\Gamma_{1}}^{\varepsilon}$, respectively, can be taken as suboptimal controls to the original problem on an $\varepsilon$-periodic square grid (8.4)-(8.8).

Acknowledgements. The authors are indebted to the unknown referees for their constructive remarks that helped considerably to improve this paper.

\section{REFERENCES}

[1] H. Attouch, Variational Convergence for Functional and Operators, Applicable Mathematics Series. Pitman, Boston-London (1984).

[2] A. Bensoussan, J.L. Lions and G. Papanicolau, Asymptotic Analysis for Periodic Structures. North-Holland, Amsterdam (1978).

[3] G. Bouchitte and I. Fragala, Homogenization of thin structures by two-scale method with respect to measures. SIAM J. Math. Anal. 32 (2001) 1198-1226.

[4] A. Braides, $\Gamma$-convergence for Beginners. Oxford University Press, Oxford (2002).

[5] G. Buttazzo, $\Gamma$-convergence and its applications to some problems in the calculus of variations, in School on Homogenization, ICTP, Trieste, September 6-17, 1993, SISSA (1994) 38-61.

[6] G. Buttazzo and G. Dal Maso, Г-convergence and optimal control problems. J. Optim. Theory Appl. 32 (1982) 385-407.

[7] J. Casado-Diaz, M. Luna-Laynez and J.D. Marin, An adaption of the multi-scale methods for the analysis of very thin reticulated structures. C. R. Acad. Sci. Paris Sér. I 332 (2001) 223-228.

[8] G. Chechkin, V. Zhikov, D. Lukkassen and A. Piatnitski, On homogenization of networks and junctions. J. Asymp. Anal. 30 (2000) 61-80.

[9] D. Cioranescu and F. Murat, A strange term coming from nowhere, in Topic in the Math. Modelling of Composit Materials, Boston, Birkhäuser, Prog. Non-linear Diff. Equ. Appl. 31 (1997) 49-93.

[10] D. Cioranescu, P. Donato and E. Zuazua, Exact boundary controllability for the wave equation in domains with small holes. J. Math. Pures Appl. 69 (1990) 1-31.

[11] C. Conca, A. Osses and J. Saint Jean Paulin, A semilinear control problem involving in homogenization. Electr. J. Diff. Equ. (2001) 109-122.

[12] G. Dal Maso, An Introduction of $\Gamma$-Convergence. Birkhäuser, Boston (1993).

[13] A. Haraux and F. Murat, Perturbations singulières et problèmes de contrôle optimal : deux cas bien posés. C. R. Acad. Sci. Paris Sér. I 297 (1983) 21-24. 
[14] A. Haraux and F. Murat, Perturbations singulières et problèmes de contrôle optimal : un cas mal posé. C. R. Acad. Sci. Paris Sér. I 297 (1983) 93-96.

[15] S. Kesavan and M. Vanninathan, L'homogénéisation d'un problème de contrôle optimal. C. R. Acad. Sci. Paris Sér. A-B 285 (1977) 441-444.

[16] S. Kesavan and J. Saint Jean Paulin, Optimal control on perforated domains. J. Math. Anal. Appl. 229 (1999) 563-586.

[17] P.I. Kogut, $S$-convergence in homogenization theory of optimal control problems. Ukrain. Matemat. Zhurnal 49 (1997) 14881498 (in Russian).

[18] P.I. Kogut and G. Leugering, Homogenization of optimal control problems in variable domains. Principle of the fictitious homogenization. Asymptotic Anal. 26 (2001) 37-72.

[19] P.I. Kogut and G. Leugering, Asymptotic analysis of state constrained semilinear optimal control problems. J. Optim. Theory Appl. 135 (2007) 301-321.

[20] P.I. Kogut and G. Leugering, Homogenization of Dirichlet optimal control problems with exact partial controllability constraints. Asymptotic Anal. 57 (2008) 229-249.

[21] P.I. Kogut and T.A. Mel'nyk, Asymptotic analysis of optimal control problems in thick multi-structures, in Generalized Solutions in Control Problems, Proceedings of the IFAC Workshop GSCP-2004, Pereslavl-Zalessky, Russia, September 21-29 (2004) 265-275.

[22] J.E. Lagnese and G. Leugering, Domain decomposition methods in optimal control of partial differential equations, International Series of Numerical Mathematics 148. Birkhäuser Verlag, Basel (2004).

[23] M. Lenczner and G. Senouci-Bereski, Homogenization of electrical networks including voltage to voltage amplifiers. Math. Meth. Appl. Sci. 9 (1999) 899-932.

[24] G. Leugering and E.J.P.G. Schmidt, On the modelling and stabilization of flows in networks of open canals. SIAM J. Contr. Opt. 41 (2002) 164-180.

[25] J.-L. Lions, Optimal Control of Systems Governed by Partial Differential Equations. Berlin, Springer-Verlag (1971).

[26] V. Mazja and A. Slutsckij, Averaging of a differential operator on thick periodic grid. Math. Nachr. 133 (1987) $107-133$.

[27] R. Orive and E. Zuazua, Finite difference approximation of homogenization for elliptic equation. Multiscale Model. Simul. 4 (2005) 36-87.

[28] G.P. Panasenko, Asymptotic solutions of the elasticity theory system of equations for lattice and skeletal structures. Russian Academy Sci. Sbornik Math. 75 (1993) 85-110.

[29] G.P. Panasenko, Homogenization of lattice-like domains. L-convergence. Reprint No. 178, Analyse numérique, Lyon SaintÉtienne (1994).

[30] T. Roubiček, Relaxation in Optimization Theory and Variational Calculus. Walter de Gruyter, Berlin, New York (1997).

[31] J. Saint Jean Paulin and D. Cioranescu, Homogenization of Reticulated Structures, Applied Mathematical Sciences 136. Springer-Verlag, Berlin-New York (1999).

[32] J. Saint Jean Paulin and H. Zoubairi, Optimal control and "strange term" for the Stokes problem in perforated domains. Portugaliac Mathematica 59 (2002) 161-178.

[33] M. Vogelius, A homogenization result for planar, polygonal networks. RAIRO Modél. Math. Anal. Numér. 25 (1991) $483-514$.

[34] V.V. Zhikov, Weighted Sobolev spaces. Sbornik: Mathematics 189 (1998) 27-58.

[35] V.V. Zhikov, On an extension of the method of two-scale convergence and its applications. Sbornik: Mathematics 191 (2000) 973-1014.

[36] V.V. Zhikov, Homogenization of elastic problems on singular structures. Izvestija: Math. 66 (2002) 299-365.

[37] V.V. Zhikov, S.M. Kozlov and O.A. Oleinik, Homogenization of Differential Operators and Integral Functionals. SpringerVerlag, Berlin (1994). 